University of Louisville

ThinkIR: The University of Louisville's Institutional Repository

Electronic Theses and Dissertations

$12-2011$

\title{
A survey study comparing adult orthodontic patient quality of life between Invisalign and fixed appliances.
}

Keith Christopher Nicholson

University of Louisville

Follow this and additional works at: https://ir.library.louisville.edu/etd

\section{Recommended Citation}

Nicholson, Keith Christopher, "A survey study comparing adult orthodontic patient quality of life between Invisalign and fixed appliances." (2011). Electronic Theses and Dissertations. Paper 1060.

https://doi.org/10.18297/etd/1060

This Master's Thesis is brought to you for free and open access by ThinkIR: The University of Louisville's Institutional Repository. It has been accepted for inclusion in Electronic Theses and Dissertations by an authorized administrator of ThinkIR: The University of Louisville's Institutional Repository. This title appears here courtesy of the author, who has retained all other copyrights. For more information, please contact thinkir@louisville.edu. 


\title{
A SURVEY STUDY COMPARING ADULT ORTHODONTIC PATIENT QUALITY OF LIFE BETWEEN INVISALIGN AND FIXED APPLIANCES
}

\author{
By \\ Keith Christopher Nicholson \\ BA, Davidson College, 2004 \\ DDS, University of North Carolina, 2008
}

\begin{abstract}
A Thesis
Submitted to the Faculty of the School of Dentistry of the University of Louisville in Partial Fulfillment of the Requirements for the Degree of

Master of Oral Biology

Department of Orthodontics University of Louisville Louisville, Kentucky
\end{abstract}

December 2011 


\title{
A SURVEY STUDY COMPARING ADULT ORTHODONTIC PATIENT QUALITY OF LIFE BETWEEN INVISALIGN AND FIXED APPLIANCES
}

\author{
By \\ Keith Christopher Nicholson \\ BA, Davidson College, 2004 \\ DDS, University of North Carolina, 2008
}

A Thesis Approved on

June 15, 2011

by the following Thesis Committee:

Thesis Director

Sunita Chandiramani, DMD MS

Eric Bednar, DDS MS

David Tasman, DMD 


\section{DEDICATION}

This dissertation is dedicated to my parents

Dr. Jack Nicholson

and

Mrs. Laura Nicholson

who have provided love and support in my endeavors and encouraged me to pursue

my personal and professional ambitions.

It is also dedicated to my brother

Dr. Eric Nicholson

who has been nothing short of an inspiration and has helped me navigate the travails of life in my feeble quest to become a complete individual. 


\section{ACKNOWLEDGMENTS}

I would like to extend an earnest thank you to my thesis director, Dr. Sunita

Chandiramani, for all of her valuable time and guidance throughout this process. I would also like to acknowledge Dr. Eric Bednar and Dr. David Tasman for their insight during this research project and thesis preparation. I would like to credit the rest of the University of Louisville faculty, staff, and co-residents who have endowed me with invaluable knowledge of orthodontics and life at large. I want to thank Dr. Dan German, Dr. Steve Burke, and staff for their participation in allowing me to use their patients for this study. Furthermore, I would like to thank Dr. Dan German for his dedication to this project, the profession, and my education. Without his tireless efforts this project simply would not have come to fruition. I want to recognize John Colgan for providing insights and volunteering his time with data collection and entry. I would also like to thank Dr. Lauren Bollenback for making me smile in a way that braces cannot. 


\section{ABSTRACT \\ A SURVEY STUDY COMPARING ADULT ORTHODONTIC PATIENT QUALITY OF LIFE BETWEEN INVISALIGN AND FIXED APPLIANCES

\author{
Keith C. Nicholson
} \\ June 15, 2011}

We aimed to examine differences in treatment impacts and quality of life between adult orthodontic patients with Invisalign and fixed appliances. Adults represent a burgeoning branch of orthodontics yet many prospective patients have been reluctant to pursue orthodontics due to concerns with treatment. It was hypothesized that removable aligners would be better tolerated by adult patients. Sixty-three adult patients (forty Invisalign, twenty-three fixed appliances) were recruited from private practice. The treatment groups were largely comparable although the braces group reported more frequent unplanned appointments and use of auxiliaries. The Invisalign group experienced less negative impacts and these differences were generally preserved after adjustment for confounding influences with the exception of pain-related impacts. The Invisalign group had a higher propensity to choose same modality again while a subjective evaluation on quality of life yielded no difference between the groups. The results may guide patient education and selection of the appropriate modality. 


\section{TABLE OF CONTENTS}

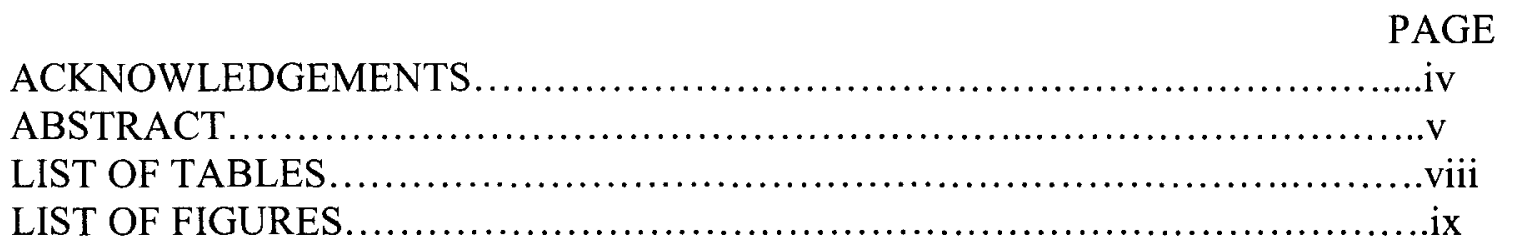

INTRODUCTION \& LITERATURE REVIEW ...................................

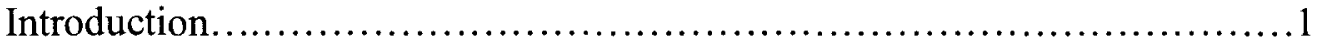

Literature Review.................................................4

METHODS AND MATERIALS.......................................... 32

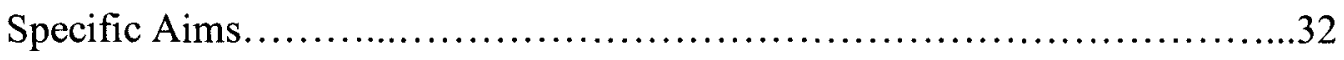

Hypotheses..................................................... 32

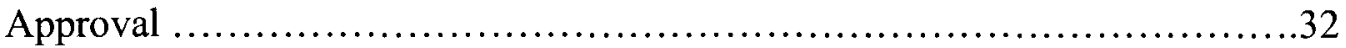

Protocol...............................................................

Inclusion/Exclusion Criteria......................................... 33

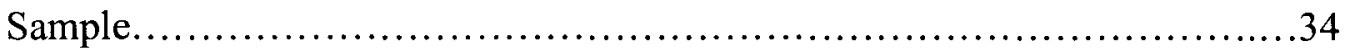

Statistical Analysis..................................................... 35

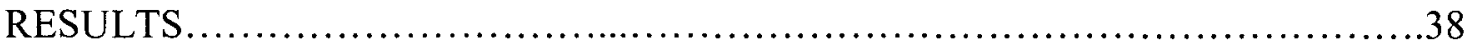

Sample Characteristics.............................................. 38

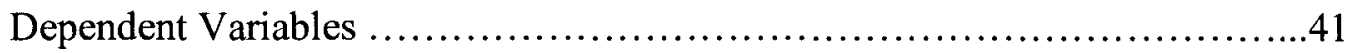

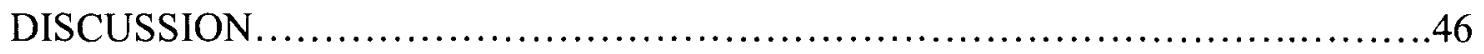

SUMMARY AND CONCLUSIONS ...........................................52

Summary........................................................52 
Conclusions........................................................52

REFERENCES ........................................................... 54

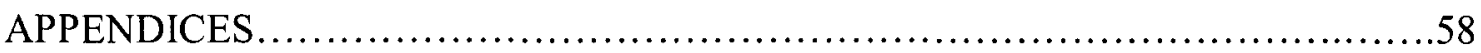

Appendix A: Survey Instrument.....................................59

Appendix B: Permission to Records....................................63

Appendix C: Informed Consent....................................64

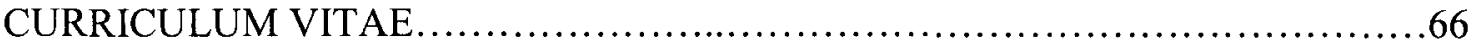




\section{LIST OF TABLES}

TABLE

PAGE

1. Survey questions with corresponding abbreviations $\ldots \ldots \ldots \ldots \ldots \ldots \ldots \ldots \ldots \ldots$

2. Distribution of demographics and other covariates..........................40

3. Outcome variables for overall sample..................................43

4. Outcome variables with regard to appliance system.........................44

5. Odds ratios (OR) for adjustment and outcome variables....................45

6. Multivariable analyses adjusted for unplanned visit and auxiliaries............46 


\section{LIST OF FIGURES}

$\begin{array}{ll}\text { FIGURES } & \text { PAGE }\end{array}$

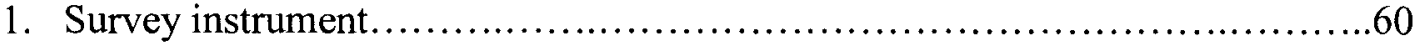

2. Primary reason for orthodontic treatment...............................40

3. Pre-treatment expectations................................................. 42 


\section{CHAPTER I}

\section{INTRODUCTION AND LITERATURE REVIEW}

\section{A. Introduction}

According to the American Association of Orthodontics (AAO), adult treatment is a burgeoning sector of orthodontics as they represent $20 \%$ of the patient population. (www.braces.org, 2011). This has grown from a paltry $5 \%$ in the 1960 s. It is reported that $56.8 \%$ and $66.3 \%$ of the adult US population exhibits crowding of the upper and lower permanent dentition, respectively (Proffit et al. 1998). Literature has suggested that adults are more critical of dental esthetics and report a higher need for orthodontic treatment than children (Stevnik et al. 1997). This authenticates adults as a significant proportion of the prospective patient pool. Furthermore, $1 \%$ of the adult population had an orthodontic appointment based on data collected from 2000-2004 (Whitesides et al. 2008). This number may grow as ostensible barriers to adult treatment are reduced.

Prospective adult patients are presented with numerous modalities for orthodontic therapy including but not limited to fixed appliances and removable thermoplastic aligners. Some prospective adult patients have previously been reluctant to pursue orthodontics in part due to compromised esthetics and discomfort during treatment (Sergl et al. 1998, Sergl et al. 200). Align Technology, Inc. (San Jose, CA, USA) formed in 1997 and has provided a clear aligner alternative to braces in correcting malocclusion since 1999. Orthodontics has previously dabbled into using aligner trays to straighten 
teeth. Kesling (1945) introduced a primordial approach to manufacture trays from plaster models making it laborious and impractical. These prototypical aligners were limited to cases with very mild treatment objectives as more complex cases required repeated impressions, models, and trays to address objectives. Invisalign revolutionized this treatment modality by introducing contemporary technology into the laboratory process (Wong 2002). It employs 3-dimensional graphic imaging, computer-aided design (CAD), and computer-aided modeling (CAM) to manufacture a series of stereolithographic (SLA) models. Subsequently, polyurethane resin trays are fabricated from the SLA models and each customized tray is worn sequentially until treatment objectives are reached. Each clear tray is designed to yield approximately $0.2 \mathrm{~mm}$ of translation and $1^{\circ}$ of rotation per tooth (Kuo et al. 2003). According to Align Technology, more than 1,000,000 patients have been treated with Invisalign to date and it is constantly evolving to improve efficiency, scope, and ease of use.

(www.invisalign.com, 2011). Case reports have been published documenting successful treatment of extraction and combined surgery-orthodontics cases (Giancotti et al. 2008, Giancotti et al. 2009, Womack 2006, Womack et al. 2008). Recently, Vardimon et al. (2010) presented a possible change to the current regimen of full time wear. Rather, a new protocol may be to wear trays full time during the first 2 days and then only part time thereafter until the next trays. Invisalign is becoming more ubiquitous as its marketing campaign has exploited many media platforms. Meier et al. (2003) showcased its pervasiveness finding that $41 \%$ of Invisalign patients cited media platforms as the initial source of information closely paralleling the $42 \%$ that reported referral from a dental professional. Align Technology has cultivated Invisalign's appeal by marketing 
directly to the consumer. The growing visibility of Invisalign warrants further investigation of this appliance system.

Barriers to adult orthodontic treatment include the visibility of appliances, discomfort, and challenges with function and hygiene (Buttke et al. 1999, Breece and Nieberg 1986, Lew 1993). Orthodontists would benefit from an appliance system that reduces any of these barriers. Invisalign system comprises the emerging paradigm of esthetically-driven orthodontic therapy. The esthetic advantage of Invisalign has been well documented and could ameliorate the social anxiety related to orthodontics (Ziuchkovski et al. 2008, Rosvall et al. 2009). The other barriers are putatively reduced with Invisalign as well but the literature is more equivocal. Many of these studies only collected data for a short segment of time relative to entire duration of treatment. Miethke et al. (2005 and 2007) demonstrated that Invisalign patients had improved plaque scores and periodontal health in comparison to fixed appliances and lingual appliances, respectively. This may curtail negative sequelae such as white spot lesions, dental caries, and periodontal disease. Invisalign generally minimizes the functional impediments associated with treatment in comparison to alternative appliances (Nedwed et al. 2005, Sergl et al. 1998). In a seminal study investigating patient tolerance of Invisalign versus fixed appliance, Miller et al. (2007) concluded that Invisalign patients experienced less pain and negative impacts associated with treatment during first week of therapy. Preliminary studies corroborate that Invisalign is more tolerable to patients. With shrewd planning, Invisalign may fulfill orthodontic goals while minimizing undesired and deleterious effects of treatment. While results are promising, the dearth of literature demands continued research to verify presumptions regarding Invisalign. 


\section{B. Literature Review}

\section{Adult Orthodontics}

\section{a. Treatment Need and Demand}

Adult orthodontics has grown substantially since the latter $20^{\text {th }}$ century. This is largely attributed to the prevalence of malocclusion as well as a reduction in the social stigma of adult orthodontics. Proffit et al. (1998) determined that nearly $2 / 3$ of adults have some form of malocclusion. Further, they determined that $15 \%$ of white children and $40 \%$ of Mexican children may reach adulthood with a moderate to definite treatment need as defined by the Index of Orthodontic Treatment Need (IOTN). A high degree of adult malocclusion is also observed in Europe. Salonen et al. (1992) examined Swedish adults and found that between $17 \%$ and $53 \%$ had malocclusion depending on which decade of life with the overall values calculated to be $35 \%$ for men and $40 \%$ for women. Burgersdijk et al. (1991) found adult malocclusion to be even more rampant among the Dutch population with an estimated $76 \%$ stricken with orthodontic problems. The pervasiveness of adult malocclusion was further corroborated by Searcy and Chisick (1994) who identified $76 \%$ of United States Army recruits as having malocclusion with nearly $16 \%$ having a severe, handicapping form. It has been firmly established that there are adults who could benefit from orthodontics. Moreover, there is evidence that adults desire improved dental appearance and function. Stevnik et al. (1997) investigated the perception of dental esthetics among a lay Norwegian population. The sample was partitioned into three groups including children, young adults, and parents and each was asked to categorize malocclusion severities. The study also served to validate the previously heralded British Aesthetic Component (AC) scale. Young adults and parents 
were generally more critical of malocclusions and were more likely to suggest orthodontic treatment. This may be intuitive but it is a testimony to the fact that adults have a more discerning eye when it comes to dental esthetics. A growing desire for orthodontic treatment is likely linked to an anticipated reward. Gazit-Rappaport et al. (2010) confirmed this suspicion in examining the psychosocial reward of adult treatment. Adult patients completed the Psychosocial Impact of Dental Aesthetics Questionnaire (PIDAQ) both pre-treatment and post-treatment serving as their own controls. Significant improvement was seen in all parameters which included dental self confidence (DSC), social impact (SI), psychological impact (PI), and aesthetic concern (AC). This conclusively shows that dental aesthetics produced a positive impact on quality of life. There is a bountiful market for adult orthodontics but it continues to be underutilized. According to Buttke and Proffit (1999), nearly $2 / 3$ to $3 / 4$ of adults have malocclusion but they only represent $15 \%$ of orthodontic patients. This has grown significantly from only $5 \%$ in 1970 to a peak of $25 \%$ in 1990 (Proffit 2007). However, there are numerous adults who could benefit from orthodontics that do not receive treatment due to barriers. Whitesides et al. (2008) summarized the socio-demographics of adult orthodontic patient. The study indicated that $1 \%$ of the adult population sought orthodontic services and the most common profile was women below the age of 30 years. When accounting for numerous covariates, no substantial racial or ethnic disparity existed.

\section{b. Barriers to Treatment}

Significant barriers to orthodontic treatment exist and are responsible for the disparity between treatment need and treatment rendered. The barriers are infinite but 
more frequent ones have been characterized in the literature. Buttke and Proffit (1999) identified several reasons for not pursuing treatment. They included lack of awareness that orthodontics can be performed on adults, fear over possible discomfort, and distress regarding social acceptance. Breece and Neiberg (1986) and Lew (1993) discovered that almost half of the prospective adult patients' treatment quoted embarrassment of appliances as the principal reason why they did not pursue treatment. However, among a sample of adults who received treatment, only $20 \%$ reported an unpleasant social impact of wearing appliances (Lew 1993). Lew (1993) reported additional roadblocks to treatment involving high cost, fear of pain, and incognizance that adults can wear appliances. Obstacles to treatment prohibit prospective patients from the positive impacts of orthodontics. If any of these hurdles can be abolished or mitigated then treatment would become more accessible.

\section{Invisalign System}

\section{a. History}

Removable orthodontic appliances rely on patient compliance but can serve as an alternative to conventional fixed appliance. Kesling (1945) introduced removable tooth positioning trays to orthodontics in the mid $-20^{\text {th }}$ century. The positioners were pliable rubber and made to fill the freeway space. His innovation was a response to the residual interproximal spaces with bands. At this time, Kesling proposed removable trays as an adjunct to fixed appliances during the finishing stages. He also recognized they could dually serve as retainers. The ultimate limitation of his primitive appliance was the onerous laboratory work required to generate positioners. Each positioner required a set of impressions, plaster models, a wax setup, and positioner fabrication. This was too 
impractical for significant tooth movements. Chained by the technology of his day, Kesling lacked the clairvoyance to see the removable modality's full potential to address comprehensive problems.

Align Technology, Inc. (San Jose, CA, USA) formed in 1997 and has provided a clear aligner alternative to braces in correcting malocclusion since 1999. Previous removable aligners could only accomplish $2-3 \mathrm{~mm}$ movement before new impressions and setup had to be performed. According to Wong (2002), Invisalign obviates this limitation by employing contemporary 3-dimensional computer-animated-design-computeranimated-manufacturing (3-D CAD-CAM) technology. Wong (2002) provides a stepwise overview of the Invisalign technology from patient records to treatment completion. Comprehensive records are collected which consist of polyvinyl siloxane impressions, a centric bite, clinical photographs, a panoramic image, and a lateral cephalogram. Additionally, the orthodontist develops a customized prescription form detailing the mechanics and objectives for the case. These items are submitted via mail or electronically to Invisalign where the impressions are, "poured up in dental plaster and then placed in a tray and encased in epoxy and urethane." (Wong 2002, pg. 540) The trays are then placed in a destructive scanner which generates a 3-D model of the upper and lower dentition. The centric bite is used to occlude the upper and lower computer renderings. From this template the virtual orthodontic technician (VOT) uses the doctor's prescription form to manipulate the models to achieve desired tooth movements. A virtual gingival is created to indicate tray borders. The technician generates a virtual setup within certain parameters set forth by Invisalign regarding the practicality and velocity of tooth movements. The setup is then submitted to the doctor who can request 
revisions until satisfied. Once approved, a series of stereolithographic (SLA) models are fabricated from the staged virtual models. Then the aligners are made on each physical model with a Biostar machine. The advent of CAD-CAM technology has transcended previous lab-intensive barriers. Kuo (2003) expounded on Wong's expansive review and broached some revisions to Invisalign's protocol. Invisalign can utilize computer tomography $(\mathrm{CT})$ for impression scanning. CT scanning is superior to laser scanning for this application because laser scanning cannot reliably perceive undercuts in impressions. With CT scanning, a series of radiographs are created as an x-ray sensor revolves 360 around impression. The library of radiographs is changed into sinograms. "A 16 centralprocessing unit fiber-optically linked computing cluster uses the sinograms and a series of mathematical algorithms to create a 116-micron thick reconstruction slices of the object. These slices are stacked electronically and inverted, and the resulting surface is smoothed to yield a raw electronic study model." (Kuo 2003, pg 579). The CT scanning method bypasses the plaster model step and improves speed and accuracy.

\section{b. Applications and Case Reports}

As with any new, tantalizing technology, there is a learning curve to understand its applications and nuances. The literature should be integrated to provide an evidencebased rubric for treatment guidelines. This provides a script for patient communication regarding expectations. Invisalign has evolved since its inception and continues to be a dynamic product. Boyd et al. (2002) were one of the first to craft guidelines for the Invisalign system. They proposed restricting to non-extraction treatment with mild crowding or spacing. They also recommended limiting treatments to fully erupted permanent dentitions, namely adults, who are capable of the compliance regimen. 
Instructions are to wear the aligners 20-22 hours per day and approximately 1-2 weeks for each tray (Boyd et al. 2002). Each tray is 0.30 " thick and allowed $0.25-0.33 \mathrm{~mm}$ movement (Boyd et al. 2002). They substantiated their recommendations by showing the successful completion of 4 cases that fit the aforementioned criteria. Boyd et al. (2002) established inchoate guidelines for Invisalign which served as a template for future treatments. Norris et al. (2002) saw the potential with incorporating Invisalign treatment into an interdisciplinary arena. They published a case report about a collaboration of orthodontics and restorative dentistry. The 39-year old male patient had a history of bruxism and his chief concerns were spacing and small, discolored teeth. Radiographic and clinical examination revealed skeletal class I, bilateral dental class I molar and cuspid, moderate upper spacing, mild lower spacing, midline deviation, upper anterior wear, loss of anterior guidance, and Bolton discrepancy (mandibular excess). An interdisciplinary approach was used to address the patient's concerns while reducing the removal of tooth structure. Invisalign was chosen as an esthetic, pre-restorative therapy. At the conclusion of Invisalign treatment, the patient's spaces were closed, midlines were improved, and bite was opened $4 \mathrm{~mm}$ to create room for veneering. The adjunctive orthodontic phase took 12 months to complete and it enabled a more esthetic and functional rehabilitation of dentition. These early reports provided some credibility for Invisalign and nurtured confidence to use Invisalign with more complex cases.

Womack (2006) treated a four-premolar extraction case exclusively with Invisalign. Womack ventured into extraction therapy by astutely beginning with an uncomplicated case. The patient was healthy and exhibited severe upper and lower crowding but no other skeletal or dental disharmonies. Four $1^{\text {st }}$ premolars were extracted 
and space closure was accomplished with group A anchorage. The canines had favorable pre-treatment distal root angulation (DRA) which produced uprighting as teeth were tipped distally. The treatment required 50 upper and 49 lower trays. Due to some anticipated challenges with excessive canine tipping during retraction the author maintained favorable pre-treatment DRA in ClinCheck TM. Additionally, vertical attachments and power arms with elastics minimized tipping side effects. The orthodontist was adept with the Invisalign system having treated 275 cases when treatment commenced. Womack advised only proficient Invisalign providers to attempt extraction cases. It is imperative to verify that trays fully seat over attachments and that treatment is tracking with ClinCheck ${ }^{\mathrm{TM}}$ projections. He also stated that Invisalign system is not effective for posterior protraction. HÖnn and GÖz (2006) similarly treated a four $1^{\text {st }}$ premolar extraction cases. The patient was a 22 -year old female with a mild Class II vertical skeletal pattern, class I molars, excess anterior overjet, and upper and lower anterior crowding with constriction. Invisalign treatment consisted of 43 maxillary and 28 mandibular trays for a total of 20 months. The treatment success rested on shrewd diagnosis in this case since it could be largely treated with tipping and mild rotations. HÖnn and GÖz (2006) acknowledge that Invisalign cannot predictably achieve bodily movement, torque, or significant rotations. Giancotti et al. (2008) illustrated that Invisalign can be used to correct deep bites. Deep bites were opened in 3 cases by controlled proclination of incisors and leveling the Curve of Spee. The treatment times ranged from 17 to 21 months with 2 out of 3 patients requiring a Case Refinement.

The budding success and diverse applications of Invisalign inspired clinicians to tackle even more challenging cases previously thought to be restricted to fixed 
appliances. Experienced clinicians recognized some latitude with Invisalign treatment when incorporating adjunctive appliances. Giancotti and Di Girolamo (2009) treated an adult patient with severe maxillary crowding despite the accepted limitations of Invisalign system. Contraindications to Invisalign therapy include space closure involving greater than $10-15^{\circ}$ mesial tipping or severe localized crowding prohibiting aligners from fully gripping teeth. To overcome these purported limitations, Giancotti and Di Girolamo (2009) staged treatment with limited, segmental fixed appliances prior to Invisalign. Upper $1^{\text {st }}$ premolars were extracted and upper canines were retracted with segmental fixed appliances using .017"x .025" TMA T-loops. Invisalign therapy began once canines were retracted. The patient wore 36 upper and 18 lower aligners followed with 9 refinements aligners, spanning a total of 23 months of Invisalign. Auxiliaries included elliptical attachments and elastics. This case embodies a crafty approach to fulfilling treatment objectives while appeasing patient's demand for esthetic appliances. Schupp et al. (2010) also employed a dynamic approach to managing complex orthodontic problems. They presented two avenues to correct a class II malocclusion with limited, preliminary appliances in conjunction with Invisalign. Case 1 was a 14-yr old female who had left unilateral dental class II. Hooks were bonded directly to upper canine and ipsilateral lower molar. The upper left segment was distalized with full time elastic wear (1/8" medium, 4oz.) in the first phase and the remaining treatment goals were addressed with Invisalign. The case finished in 23 months with Invisalign and elastics. Case 2 was a 14-yr old male with bilateral dental class II malocclusion. A preemptive phase of distalization was accomplished with the Carrière Distalizer. This appliance stabilizes the posterior segment to be distalized and the force system includes 
elastics. These mechanics can be intramaxillary anchored to a temporary anchorage device (TAD) or intermaxillary anchored to the ipsilateral opposing arch. This requires nearly full time elastic wear. The patient's anteroposterior malocclusion was nearly corrected in 4 months so Invisalign treatment was implemented at this stage. The total treatment time was 14 months. A creative approach to class II orthodontic therapy with Invisalign and auxiliaries allowed for expedient treatment and positive outcomes sans conventional brackets.

With careful planning, Womack and Day (2008) introduced the possibility of surgical-orthodontic treatment with Invisalign. The patient was a 37-year old male with a Class II division 2 malocclusion. He had a history of snoring and disrupted sleep but no documented sleep apnea studies were performed. Pre-surgery goals were alignment and slight advancement of upper anterior teeth, leaving Iowa spaces. The surgical plan was maxillo-mandibular advancement. Specifically, this included a 2-piece Lefort advancement and expansion, and mandibular advancement with chin augmentation. Presurgical orthodontic treatment included 22 upper and 13 lower aligners over course of 8 months. After 6 weeks of retention, the bimaxillary surgery was performed. Upon anesthesia, upper and lower fracture bars were placed for post-surgery intermaxillary elastics. Then bilateral sagittal split osteotomies (BSSO) were performed using maxillomandibular fixation (MMF) and a prefabricated splint as a guide. The maxilla was subsequently advanced into class I and expanded with a 2-piece Lefort. The movements were stabilized with a palatal soft-tissue splint and rigid fixation. After several weeks of intermaxillary elastics to fracture bars, Invisalign trays were reintroduced. The post-surgical refinement required 15 upper and 6 lower trays 
amounting to 6 months of additional treatment. With meticulous planning and proper case selection, surgical-orthodontic cases can be treated to achieve skeletal harmony, functional occlusion, and esthetic improvement.

\section{c. Efficacy and Clinical Trials}

Exuberance for a new technology should be contained and subject to the same scrutiny as conventional appliances. While Invisalign is adaptive and has refined its product, it has not traversed all limitations. It is still a nascent technology that is not a remedy to all orthodontic problems. Beyond case reports, Invisalign performance in more objective studies has been irresolute. This underscores the need for appropriate case selection. Kravitz et al. (2008) identified some limitations with canine derotation. Invisalign had putatively overcome this limitation with the inclusion of vertical attachments and interproximal reduction. Kravitz et al. conducted a prospective clinical study that tested the efficacy of attachments or interproximal reduction in successfully derotating teeth as predicted by virtual models. "The derotation of cylindrical teeth presents a biomechanical challenge due to the lack of interproximal undercuts causing the aligner to slip as it attempts to rotate the tooth" (Kravitz et al. 2008, pg 682). The sample had 31 patients with a total of 53 canines evaluated ( 33 maxillary, 20 mandibular). The sample was partitioned into 3 groups based on the treatment approach to correct rotation. They were attachments only (AO), interproximal reduction only (IO), and a control with neither attachments nor interproximal reduction $(\mathrm{N})$. Pre-treatment virtual models of the anticipated outcome were superimposed on the actual post-treatment virtual models using Invisalign's ToothMeasure software. The mean accuracy for the entire sample was a modest $35.8 \%$. Using one-way analysis of variance (ANOVA) $(p<.05)$, there was no 
statistical difference between any of the treatment groups. Clinicians must carefully evaluate the authenticity of projected tooth movements. Kravitz et al. (2009) later used ToothMeasure superimpositions to conduct a prospective clinical trial on the efficacy of anterior tooth movement with Invisalign. They compared anticipated tooth movement on pretreatment virtual models of final outcome versus the actual results. The sample consisted of 37 patients consecutively treated with anterior Invisalign. This sample included 401 anterior teeth (198 maxillary, 203 mandibular). The mean accuracy was $41 \%$ ranging from $29.6 \%$ for extrusion to $47.1 \%$ for lingual constriction. There was no statistical difference between maxillary and mandibular teeth. In accordance with the previous study on canine rotations, all rotations greater than $15^{\circ}$ resulted in a drastic decrease in efficacy. Vincent (2005) evaluated Invisalign outcomes with the American Board of Orthodontics (ABO) objective grading scores (OGS). It was a retrospective study comparing pre-treatment and post-treatment dental casts as evaluated by OGS alignment and occlusion values. A total of 65 patients from 7 private practices were treated exclusively with Invisalign. Treatment time was, on average, 12.5 months. The post-treatment OGS values were statistically lower than pre-treatment values with a mean improvement of 4 points. The best results manifested with alignment and interproximal space closure while posterior occlusal contacts actually worsened. The latter is often seen immediately following Invisalign treatment due to tray thickness and may be partially resolved with a short course of posterior vertical elastics to clear attachments. The mixed results require future research concerning the biomechanics of the Invisalign. Overcorrection has been proposed as a possible remedy. Invisalign has dedicated time to improving treatment mechanics but some innovations have not been tested clinically. 
Shrewd clinicians must identify these shortcomings at the onset and factor them into treatment planning.

There is a paucity of clinical studies using Invisalign. Lagravère and Flores-Mir (2005) uncovered this by their disappointing conclusions from an attempted systematic review of Invisalign treatment effects in non-growing patients. The authors searched numerous electronic databases with "Invisalign" as the only term. They further reduced the search to human clinical trials regarding Invisalign treatment effects. This produced a paltry 2 studies. Upon further review, the authors determined that both studies were plagued with methodological issues and yielded only a low level of evidence (level II). No veritable conclusions can be drawn from the scarcity of literature on Invisalign treatment efficacy. It was interesting to note that the dropout rates of the 2 trials were $5 \%$ and $71 \%$. Djeu et al. (2005) recognized this deficiency of trials so they performed a retrospective, cohort study that compared outcomes of Invisalign versus traditional fixed appliances (control). The sample was extracted from an ABO certified orthodontist and segregated in two groups, each with 48 patients. The groups were matched using the discrepancy index (DI) and then compared post-treatment with OGS values. There was no statistical difference in pretreatment DI scores among groups. The mean DI scores for Invisalign and control were 18.67 and 19.85, respectively. ABO guidelines would rank these as moderate complexity. Following treatment, the Wilcoxon 2-sample test indicated that control OGS values were significantly lower than the Invisalign OGS values by $27 \%$. The mean OGS values were -43.35 and -32.21 for Invisalign and control, respectively. It should be noted that cases penalized 30 OGS points or fewer are typically approved by the ABO Phase III examiners. The results were further divided into 
categories representing different types of movements. This showed that Invisalign had statistically underperformed controls in obtaining ideal buccolingual inclination, occlusal contacts, occlusal relations, and overjet. Some further considerations are that the clinician who conducted treatment was far more experienced with fixed appliances. The control group benefited from the use of auxiliaries while the Invisalign group did not. This could influence outcomes, especially overjet. Additionally, final records were taken immediately after treatment completion and Invisalign cases often finish with mild posterior open bite prior to settling due to tray thickness. The Invisalign group was, on average, in treatment 3-4 months less than controls. This study established some important concerns with Invisalign system. However, it is duly noted that some shortcomings of study may bias to results.

While few robust conclusions can be derived from current clinical trials regarding treatment outcomes, contemporary research has unveiled new information about the biomechanics of Invisalign and it has implications for the tray regimen. Vardimon et al. (2010) conducted a prospective, cohort study examining in-vivo von Mises strains to better understand Invisalign force systems. Forces are related to the strains on aligner surfaces so von Mises strains delineate orthodontic forces. The von Mises strain is a theoretical value characterizing principal strains and it is formulated from distortion energy. Strain develops in trays due to the resistance to dental movement generated by the periodontal ligament (PDL) and alveolar bone. As the tooth is displaced in the PDL, tray deformation decreases and vice versa. Thus, tray deformation can quantify the amount of tooth displacement. Since the PDL thickness in the cervical and apical regions exceeds that in the middle, the $0.2 \mathrm{~mm}$ of tooth displacement per tray generally produces 
tipping. The aim was to descry the influence of time and location on strain distribution in Invisalign trays. The sample included 3 patients (adult male, adult female, adolescent male) with excessive overjet using upper and lower Invisalign trays to retract maxillary anterior teeth with maximum anchorage. Measurements were taken on days 1, 2, 9, and 15 for each set of maxillary aligners (total of 61 for 3 subjects) where subjects switched trays biweekly. For each patient a duplicate set of aligners was fabricated with 2 strain gauge rosettes bonded on the vestibular side of tray ( 1 on maxillary central incisor, 1 on maxillary premolar). These analog trays were inserted at each measurement interval and used to calculate in-vivo strains. One limitation is that the measurements do not account for intraoral aging of trays. In all subjects, peak incisor active unit strains (IVM) developed at day 1 and decreased at day 2 where they plateaued for the remainder of treatment. This indicates that most tooth movement transpires in the first day. The premolar active unit strain (PVM) exceeded the IVM in one subject implying anchorage loss. This beckons the need for attachments to maximize anchorage in such cases. As treatment progressed, the peak IVM strains increased with each sequential aligner. This may warrant increasing either the thickness of final aligners or the length of time the final aligners are worn. This study provided novel insight regarding the force systems of Invisalign trays and may sanction changes in treatment design and regimen. It is plausible that the patients would only need to wear trays for 22 hours per day during the first 2 days and then decrease to 12 hours per day until the next set of aligners. While the precise regimen cannot be inferred from this study the general concept may promote improved patient acceptance of orthodontic therapy with Invisalign.

\section{d. Invisalign Patient Profile}


Given the documented functionality of the Invisalign system and the growing sector of adult patients, Invisalign has become ubiquitous in adult orthodontics as more than 1,000,000 patients have been treated with Invisalign to date (Align Technology, Inc. www.invisalign.com). This is partially responsible to its ability to mitigate barriers that previously discouraged adult patients. Additionally, Align technology, Inc. has made their product visible by exploiting marketing platforms such as social media.

Recognizing its impact on orthodontics, Meier et al. (2003) sought to characterize the Invisalign patient profile with a prospective study assembling some personal data and clinical findings of patients interested in Invisalign therapy. The Department of Orthodontics and Dentofacial Orthopedics, Charité Medical School, Humboldt University, Berlin offered special consulting hours to prospective Invisalign patients. Of the 301 patients who received consultation, 89 completed a voluntary survey and underwent a clinical examination. The survey was expansive and recorded information such as demographics, treatment goals, tolerable treatment times, and source of referral. Females represented $72 \%$ of the 89 participants. The mean age was 35 years with a range of 15-68 years. The most common gender and age group (decade intervals) was females between the ages of 20 to 29 years. Most patients would accept treatment lasting 1.5 to 2.5 years. The primary treatment objective was esthetic improvement which embodied $97 \%$ of the sample. Esthetic concerns were also evident in the fact that $62 \%$ rejected treatment with visible appliance. The most prevalent dental findings were anterior crowding at $87 \%$, midline deviations at $63 \%$, and class II dental relationship at $49 \%$. The frequency of anterior crowding is congruous with esthetic concerns. Of particular interest was the source of information regarding Invisalign treatment. The plurality was 
informed by a dental professional (42\%) but an almost equal number (41\%) first discovered Invisalign through social media (press, television, Internet). This reveals the pervasiveness of Invisalign in society and reflects a campaign to market directly to the consumer.

\section{Esthetics}

Invisalign has purported advantages compared to alternative appliances such as improved esthetics, improved hygiene, and improved patient tolerance. Some of these relate to the obstacles to adult orthodontic treatment previously discussed (Buttke and Proffit 1999, Breece and Neiberg 1986, Lew 1993). Improved esthetics and hygiene have been well been documented in previous studies whereas improved patient tolerance of treatment is multifactorial and the current support is untenable. All three marketed advantages will be investigated in this study with the focus on possible improvement with patient tolerance.

Invisalign fits the mold of the esthetic paradigm in orthodontic appliances.

Numerous survey studies have demonstrated that removable aligners are less conspicuous than other appliances and thus are more esthetic. Ziuchkovski et al. (2008) assessed the attractiveness of orthodontic different appliances with a computer-based survey. The relative esthetics among different appliance types, brands, wires, and ligature ties were tested. The same model was used for all images to control for outside variables influencing survey responses. Photoshop (version 7.0; Adobe) was used to standardize the pictures for color and format. When applicable, bracket placement was standardized with pre-fabricated placement jigs. The survey contained two yes-no questions regarding acceptability of appliance as well as a visual analog scale (VAS) to rate attractiveness of 
appliance. A composite of six appliance images was shown initially to the subjects for calibration. Then each subject scrolled through nine images which included three repeats to verify intraexaminer reliability. The sample consisted of 200 subjects with $88.8 \%$ having no formal dental training and $11.2 \%$ having some dental experience. For all demographics, the hierarchy of attractiveness was alternative (clear trays or lingual) > ceramic $>$ self-ligating / steel appliances. There was no difference between brands. Wire and ligature ties had some effect with ceramic brackets but no influence with steel appliances. Clear trays and lingual braces are unequivocally more esthetic than other appliances. Rosvall et al. (2009) reaffirmed many of these conclusions with a corollary survey study. A computer-based survey was used to quantify the laypersons' perspective on attractiveness, acceptability, and monetary value of various orthodontic appliances. The same methods were used as in the Ziuchkovski et al. (2008) study. This survey was amended to include questions regarding the additional value of alternative appliances in comparison to steel appliances. The sample was comprised of 50 adult subjects with no formal dental training. The findings on esthetics and acceptability in relation to various orthodontic appliances are congruous with previous results. The hierarchy was alternative appliances (clear trays or lingual braces) $>$ ceramic $>$ ceramic self-ligating $>$ hybrid self-ligating stainless steel and self-ligating stainless steel. This is substantiated with the acceptability results in which over $90 \%$ find alternate and ceramic appliances to be acceptable. To the contrary, only $55 \%$ and $58 \%$ felt the traditional stainless steel and self-ligating stainless steel were acceptable, respectively. Interestingly, there was no difference in response regarding acceptability for themselves or for their children. The mean willingness to pay (WTP) values indicate that subjects will pay a $\$ 610$ premium for 
alternative appliances, $\$ 329$ for ceramic traditional and self-ligating appliances, and $\$ 167$ for hybrid self-ligating appliances. These pioneering studies certify the importance of esthetic treatment modalities.

\section{Periodontal Health}

Orthodontic treatment has been associated with deleterious effects to the periodontium precipitated by inadequate hygiene. Hygiene is more challenging with orthodontic appliances as they present a physical barrier to brushing and they can serve as trap for food and plaque. Plaque contributes to sequelae such as decalcifications and periodontal disease. The latter is even further exacerbated in the presence of orthodontic forces. Thus, measures have been taken to reduce these potential detrimental effects during orthodontic treatment. One possible remedy is an appliance system that does not interfere with plaque removal. Invisalign intuitively fits this criterion because the trays can be removed during eating and hygiene. However, this needs to be demonstrated in clinical trials since there are numerous variables that contribute to the development of disease.

Lingual appliances are the principal competitor of Invisalign in the field of esthetic orthodontics. Therefore, it is reasonable to evaluate lingual appliances' impact on oral health and function. Hohoff et al. (2003) conducted a prospective, longitudinal study on the oral comfort, function, and hygiene in patients with lingual brackets. Previous studies had been largely limited to retrospective analysis. The sample comprised 22 adults ( 5 male, 17 female) extracted from consecutively treated maxillary lingual orthodontic patients using the Ormco $7^{\text {th }}$ generation lingual bracket. Each proband completed a survey questionnaire at 3 different time intervals including pre- 
treatment, within the $1^{\text {st }}$ day of treatment, and 3 months later. The questionnaire consisted of 10 questions related to oral comfort, function, and hygiene. If a patient reported poor scores in a given category prior to treatment then that particular parameter was excluded from data analysis. Compared to pretreatment, significantly poorer responses were seen at both time intervals during treatment. Conversely, significant improvements were seen in some parameters between the two treatment intervals which can be, in part, attributed to adaption. Items that failed to improve were tongue space, tongue position, chewing, biting, and oral hygiene. The patient response was noticeably worse than previously recorded in retrospective studies. The results are edifying and reveal that oral function and comfort can be afflicted by lingual appliances.

The periodontal response to Invisalign treatment was evaluated in comparison to conventional fixed appliances and separately to lingual appliances in two corollary studies. Miethke and Vogt (2005) conducted a prospective clinical trial and pilot study to examine the periodontal impact of Invisalign versus traditional braces. The sample comprised two groups of 30 (60 total) consecutively treated patients with either Invisalign or fixed appliances at the Department of Orthodontics and Dentofacial Orthopedics of the Charité Berlin clinic. The patients' periodontal health was measured with 4 parameters including Gingival Index (GI), modified Plaque Index (PI), modified Papillary Bleeding Index (PBI), and sulcus probing depth (SPD). These were recorded at 3 time intervals during treatment. The patients were instructed to maintain current oral hygiene practices prior to the first recording and were subsequently given oral hygiene instructions $(\mathrm{OHI})$ for remainder of the trial. The Invisalign subjects had significantly lower PI scores in totality over the 3 intervals but the most pronounced difference was at 
the first evaluation. There was no statistical difference between the groups with any other parameters. Both groups improved scores over course of study, likely in response to specific OHI. Invisalign enables easier plaque removal but may not fully alleviate the challenge in maintaining a healthy periodontium. This study would have benefited from a control without orthodontic appliances. Miethke and Brauner (2007) performed a corollary clinical trial investigating periodontal health during treatment between competing esthetic modalities, Invisalign and fixed lingual appliances. It was similar in design to the previous study by Miethke and Vogt (2005). The Invisalign group was the same 30 patients used in previous study. The fixed lingual appliances group consisted of 30 consecutive patients recruited from the Department of Orthodontics and Dentofacial Orthopedics of the Charite Berlin clinic as well as two private practices. Of the four periodontal parameters used, all but SPD was significantly better in the Invisalign group. Another salient trend was that the Invisalign group improved scores as treatment progressed, likely a reflection of $\mathrm{OHI}$ once treatment commenced. However, the lingual fixed appliance group did not show appreciable change during the course of study despite thorough OHI. This suggests that lingual appliances may pose a difficultly to hygiene regardless of homecare regimen. At this stage, it can be concluded that Invisalign is a more biologically compatible with the periodontium.

\section{Appliance Acceptance and Patient Tolerance}

Orthodontists endeavor to use an appliance system that predictably accomplishes treatment objectives and is most accepted by patients. The published efficacy of Invisalign has already been reviewed. While removable aligners are more esthetic and may facilitate improved periodontal health, it is spurious to conclude that they are better 
tolerated by patients. Rather, survey studies and clinical trials have the burden of establishing which modalities provide the best experience for orthodontic patients during active treatment.

Lingual appliances have offered patients an esthetic alternative to traditional braces. However, they can pose a difficulty to patients with regards to hygiene and comfort. Fritz et al. (2002) conducted a retrospective, survey study about the lingual technique throughout the entire course of treatment. They characterized the patient profile, motivation, and acceptance. Participants were consecutively treated patients from a private practice in Bad Essen, Germany and the Orthodontic Clinic, University of Aachen, Germany. The inclusion criteria required completion of comprehensive orthodontics with fixed lingual appliances in the last 6 months. Surveys were distributed to 110 subjects and 98 responded yielding a dropout rate of $11 \%$. Relocation is the primary reason cited for dropouts albeit this is conjecture. The comprehensive questionnaire covered information about demographics and treatment parameters such as motivation, source of information, treatment time, phonetic-functional impairments, satisfaction with result, and general acceptance of appliance. The principal sources of information were friends and dental professionals representing $93 \%$ of sample. This departs from the Invisalign profile in which nearly half the patients discovered Invisalign via media platforms (Meier et al. 2003). The preponderance of subjects was females below the age of 40 years whose cardinal motivation for the lingual technique was esthetic concerns. Almost half the sample (47\%) quoted moderate impairments related to lingual appliances. This is significant but almost none answered with severe impairments. The phonetic-functional adaptation was reported to be between 1 to 3 
weeks by $65 \%$. The overall satisfaction with treatment was positive with $99 \%$ at least content with outcomes and $87 \%$ indicating they would recommend lingual orthodontics to others. These results were more favorable than previous studies. One plausible explanation is that $95 \%$ reported being adequately educated about anticipated issues. Thorough discourse may assuage patients regarding future problems. Excitement should be guarded though considering the relatively high dropout rate $(11 \%)$ which may reflect dissatisfied patients. Hohoff et al. (2003) studied speech performance in lingual patients with a prospective, longitudinal trial. The sample included 23 patients $(6$ men, 17 women) with a mean age of 35.1 years. Patients with a previous history of speech impairments were excluded. The patients were evaluated at 3 intervals, including pretreatment, within 24 hours of bonding, and 3 months into treatment. Speech was evaluated by a myriad of articulation tests involving objective digital sonography, semiobjective examination by professionals, semiobjective evaluation by close contacts of patient, and subjective report by patient. All testing methods yielded a significant deterioration at both treatment intervals in comparison to pre-treatment scores. However, a mild improvement (adaptation) was reported in some reports. There was no gender impact. These findings warrant thorough patient education regarding the impact on speech. While an appliance might be inconspicuous by sight, it may be conspicuous by its auditory impact.

Previous studies have catalogued the performance of various appliance systems regarding discomfort, compliance, and patient acceptance. While some used a precursor to Invisalign as the removable modality, they provide some fundamentals for understanding the differences between patient quality of life with fixed and removable 
orthodontics. Stewart et al. (1997) investigated the patient's perspective of appliance wear. The sample contained 52 consecutive patients selected from the Glasgow dental school and subsequently divided into two groups, comprehensive fixed appliances and maxillary removable plate, based on which modality was used to treat their malocclusion. Of the 52 subjects, 17 were males and 35 were females with an age range of 9 to 30 years. Each subject completed a survey questionnaire pertaining to comfort, convenience, and self-consciousness using a 4-point Likert scale. The survey was filled out daily for the first week and then again at 2 weeks and 3 months. The patients went to monthly recalls for adjustments. The most deleterious effects concerning tightness and sensitivity were associated with fixed appliances on day 1. Most problems with discomfort waned after 4-7 days in both groups. Improvement was seen throughout duration in nearly all categories for both groups. The problems with fixed appliances were generally more pronounced than with the removable group. However, speech and swallowing were more adversely affected with the removable plate and this persisted to a small extent. Interesting to note is that self-consciousness regarding appliance wear in public was initially worse with the fixed group but ultimately abated to the same level as the removable group. This information should guide clinicians in educating patients of the initial impact and likely adaptation with each appliance. This sample contained mostly adolescents so adaptation may be different with adults. Sergl et al. (1998) ran a survey study about how appliance type and patients' perception of their malocclusion severity affect patient discomfort, acceptance, and compliance. A total of 84 (39 males, 45 females) orthodontic patients with a mean age of 12.8 years completed questionnaires. The treatment groups included comprehensive fixed appliances, functional appliance, 
upper and lower removable plates, and an upper removable plate. The surveys were completed daily for 1 week and then again at 2 weeks, 3 months, and 6 months. Patients with fixed or functional appliances had significantly worse scores in reference to discomfort, although pain was mollified in all cohorts within the first week. Patients who perceived their malocclusion as more severe had significantly lower pain scores indicating they might better tolerate discomfort. Both acceptance and compliance were negatively related to the intensity of discomfort reports. This was a multifactorial approach to understanding issues related to discomfort, acceptance, and compliance of orthodontic patients. Sergl et al. (2000) followed up with previous study (Sergl et al. 1998) to research the functional and social discomfort associated with orthodontics. The survey also inspected how personality variables influence compliance and adaptation. This follow-up investigation used the same sample as Sergl et al. (1998). Collectively among all appliance types, there was a significant reduction in discomfort during the first week and subsequently thereafter. Interestingly, the severity of discomfort was not decidedly related to the time intervals, implying it may have more to do with personality characteristics. There was no significant relationship between social apprehension and the type of appliance type. Functional appliances and bimaxillary removable plates yielded a slight negative correlation with speech and swallowing. The subjects' perceived severity of malocclusion was inversely related to reports of discomfort during treatment. An index used to quantify subjects value on dental esthetics had a significantly negative relation to discomfort during treatment as well. All complaints were inversely related to appliance acceptance for all groups. This study characterized 
some basic trends evident with orthodontic appliances and discomfort, adaptation, and acceptance.

Contemporary studies have continued to explain the discomfort and treatment impacts of Invisalign therapy. Schaefer and Braumann (2010) administered a prospective, crossover study evaluating halitosis, oral health, and quality of life during Invisalign treatment with and without chlorhexidine (CHX). The sample was 31 adult Invisalign patients ( 7 male, 24 female) and they were evaluated during the first 8 months of Invisalign treatment. A cross-over design was used whereby patients were partitioned into 2 groups, (group 1: $\mathrm{CHX} /$ no $\mathrm{CHX}$, group 2: no $\mathrm{CHX} / \mathrm{CHX}$ ). The two study periods were 3 months long with 2 months of wash out between periods. Invisalign therapy was continued without disruption during the 8 months. Surveys and measurements concerning halitosis, oral health, and quality of life were collected at numerous intervals during the 8 months. There was no evidence of halitosis, dry mouth, increased inflammation, or elevated plaque indices during Invisalign therapy. Invisalign exhibited a mild impact on quality of life associated with oral health. These ranged from impacts on speech, diet, discomfort, and anxiety. The results are edifying but there was no control group and the study period was limited to the first 8 months of treatment. Nedwed and Miethke (2005) designed a survey study to evaluate motivation, acceptance, and problems with Invisalign patients. The sample was derived from the first 54 (14 males, 40 females) Invisalign patients treated at the Department of Orthodontics and Dentofacial Orthopedics at Charité. The mean age was 33 years old. Each subject completed a 12-question survey questionnaire 3 to 6 months into treatment regarding motivation and acceptance of aligners. Specifically, it examined adaptation time, pain, 
speech, mucosal irritations, mastication, temporomandibular dysfunction (TMD), and subjective evaluation on the success of treatment. The results were propitious for Invisalign treatment. Almost all subjects (83\%) adapted to Invisalign within 1 week and experienced only mild (54\%) or no pain (35\%) during treatment. As with alternative modalities, peak pain was experienced within 2 to 3 days after adjustments. Most patients reported no speech impediment (93\%), no narrowing of lingual space (76\%), no mucosal irritations ( $70 \%)$, and no TMD (92\%). Of the small $8 \%$ TMD patients, their signs were limited to clicking which was present prior to treatment in all cases. Most patients $(89 \%)$ were satisfied with treatment progress at the time of survey. The one inauspicious finding was that $44 \%$ had difficulty chewing, citing tooth sensitivity and interproximal spaces as primary culprits. These results pale into comparison to the adaptation time and problems reported with other modalities, especially lingual appliances. As with the Schaefer and Braumann (2010) crossover trial, this survey only followed patients for a portion of active treatment. It was also weakened by the lack of a control group. Miller et al. (2007) conducted a prospective, longitudinal cohort study comparing treatment impacts between Invisalign and fixed appliance therapy during the first week of treatment. The study contained 60 adult patients treated with Invisalign or fixed appliances. Each treatment group had 30 subjects who completed a survey questionnaire daily for the first week of treatment. The daily log included demographic information and 3 sections pertaining to treatment impacts. The Geriatric Oral Health Assessment Index (GOHAI) was used as a template in survey design (Atchison and Dolan 1990). The three sections included a visual analog scale (VAS) for pain severity, an item inquiring about medications to manage discomfort, and 13 questions using the 5- 
point Likert scale to characterize functional, psychosocial, and pain-related impacts. The two treatment groups were matched in terms of sex, race, education, health status, previous treatment, and Peer Assessment Rating (PAR) index. Significant differences existed between the groups regarding income, reason for treatment, and age. The Invisalign group had a statistically higher income and older age. The age difference may be trivial since all patients were adults. More Invisalign patients sought treatment for improved appearance ( $85 \%$ versus $67 \%$ ), while the fixed appliances patients sought treatment more frequently by dentist referral $(26 \%$ versus $3 \%)$. The baseline values for both groups were equivalent. The overall treatment impacts for the entire week were significantly less negative for the Invisalign patients in each of the 3 subscales (functional, psychosocial, pain-related). The Invisalign group saw mean values in each subscale drop to baseline values by the end of the week whereas the fixed appliances group only returned to baseline values in the psychosocial subscale. The mean VAS scores were significantly lower (less severe pain) for the Invisalign group over the course of the week. The Invisalign VAS values returned to baseline level by day 5 whereas the fixed appliance group remained above baseline at day 7 . Both groups reported only taking over-the-counter (OTC) medications to manage pain. There was no significant difference at baseline, day 1 , or days 4 through 7 . The fixed appliances subjects took significantly more medications on days 2 and 3 . These results suggest that the negative treatment impacts associated with orthodontic therapy are significantly milder with Invisalign than with fixed appliances. It also contends that patients adapt more quickly to Invisalign trays. While this study controlled for most covariates there were some differences in income, age, and reason for treatment. Additionally, it only represents 
impacts during the first week of treatment. These 3 studies summarizing discomfort and treatment impacts illustrate that Invisalign trays perform well, incite minimal discomfort, and produce little interference with oral function. However, there is a need for corollary studies utilizing a control group and collecting patient data referencing the entire duration of treatment. 


\section{CHAPTER II}

\section{METHODS AND MATERIALS}

\section{A. Specific Aims}

$\mathbf{1}^{\text {st }}$ Aim: Examine if there is a difference in negative impacts experienced by adult patients during the entire course of orthodontic treatment among two different treatment modalities, Invisalign and traditional fixed appliances.

$2^{\text {nd }}$ Aim: Test if there is a difference in subjective adult patient quality of life during the entire course of orthodontic treatment among two different treatment modalities, Invisalign and traditional fixed appliances.

$\mathbf{3}^{\text {rd }}$ Aim: Test if there is a difference in subjective adult patient satisfaction related to treatment modality among Invisalign and traditional fixed appliances as measured by the predilection to choose the same system again if granted the choice.

\section{B. Hypotheses}

1. Research Hypotheses

The Invisalign group will report less negative impacts associated with orthodontic treatment and will tolerate orthodontic treatment better than control group.

2. Null Hypotheses

There will be no difference in the severity of negative impacts or the subjective patient quality of life reports among the two appliance systems, Invisalign and fixed appliances.

\section{Approval}


This study was conducted in accordance with Internal Review Board (IRB) guidelines. It received IRB approval on 1/10/2011 and it expires on 1/9/2012. The University of Louisville IRB tracking number is 11.0002 .

\section{Protocol}

The present study was a cohort study that employed a survey instrument (See Figure 1 in Appendix A) to extract information about orthodontic treatment impacts and subjective tolerance of appliances. The survey design used the Geriatric Oral Health Assessment Index (GOHAI) as a template and was amended to include questions germane to treatment impacts and covariates. GOHAI is cited in the literature as a veritable index of patient satisfaction. Additional questions were included to address the specific aims of the present study and account for possible covariates. The survey questions primarily utilized the 5-point Likert scale. Questions with integer answers were limited given concerns with reliability since responses were based on recall. Otherwise data included categorical and interval variables.

\section{E. Inclusion/Exclusion Criteria}

Inclusion Criteria:

1. Comprehensive orthodontic treatment.

2. At least 18 years old when treatment commenced.

3. Treatment completed within past 2 years.

Exclusion Criteria:

1. Hybrid treatment involving both Invisalign and fixed appliances during most recent phase of orthodontics.

2. Major health ailments that significantly affected activities of daily living (ADLs) 


\section{F. Sample}

Patients who fulfilled aforementioned criteria were identified in two private practice offices. The private practitioners were Drs. Daniel German and Stephen Burke of Beavercreek, $\mathrm{OH}$ and Huber Heights, $\mathrm{OH}$, respectively (See Appendix B). Each practitioner has more than 15 years of clinical experience and both are Premier Invisalign providers. Premier providers were purposely selected to reduce potential bias concerning experience with the two appliance systems. Eligible patients were approached about voluntary participation in study. The methods of contact were phone calls, mailings, and in person during debond or retention appointments. Surveys were mailed or given to patient during office visit. Each prospective subject was provided with the study preamble (See Appendix C), survey, and an addressed University envelope with paid postage. The surveys were completed on subjects' own volition and sent directly to the University of Louisville Department of Orthodontics. A patient coding system was developed to ensure there were no identifiers that would violate anonymity in accordance with IRB.

A total of 74 adult patients returned surveys. Sample attrition was 11 patients due to incomplete surveys, inability to identify patients, or failure to meet study criteria as delineated above. A total of 63 subjects were used for study purposes [Invisalign: 40, Fixed appliances (control): 23]. The sample comprised 48 females and 15 males. The mean and median ages of the total sample were 40.4 and 42 years, respectively. Table 2 characterizes the sample in regards to demographic variables and other possible adjustment variables. This data was numeric, categorical, and interval-based. These variables were analyzed to control for potential impact on dependent variables. 


\section{G. Statistical Analysis}

For analyses involving an association of categorical and interval variables with appliance system, Pearson's chi-squared test or Fisher's exact test were used to calculate p-values. Variable levels with less than 5 counts were excluded from statistical analysis. For testing differences in the central tendencies of numeric outcome variables between the two treatment groups, the t-test or Wilcoxon Rank Sum test were used. All scales were considered to be numeric data for statistical purposes. Variables were considered to be significant at the $\alpha=0.05$ level. The outcome variables were compared between the treatment groups. For confounding variables that exhibit a statistically significant difference among treatment groups, a multivariable analysis was conducted a posteriori to evaluate consequence on outcome variables.

Each question from the survey was designated an outcome variable or covariate. The outcome variables correlate with the specific aims of the study. The statistics identify whether or not a difference exists between the treatment groups in regards to 6 outcome variables. The outcome variables comprise 4 interval variables derived from question 9, a subjective nominal variable pertaining to question 17 , and a numeric variable corresponding to question 19. The 4 analyses procured from question 9 were semi-objective measures that quantify the negative impacts associated with appliances. Each response is scored in the 5-point Likert scale and high scores indicate less negative impacts. The sum of these scores were used for analysis and considered to be numeric data for statistical purposes. Questions $9 \mathrm{e}$ and 91 were modified since higher scores implied more negative impacts. As a result, the responses were inverted to be congruent with other responses. The analyses extracted from question 9 define the overall impact as 
well as 3 subcomponents which relate to the functional, psychosocial, and pain-related impacts. The collective impacts were calculated by summing all 13 responses $(9 \mathrm{a}-\mathrm{m})$ while the subscales for functional, psychosocial, and pain were computed by adding responses from $9 \mathrm{a}-\mathrm{d}, 9 \mathrm{e}-\mathrm{i}$, and $9 \mathrm{j}-\mathrm{m}$, respectively. The subjective variable from question 17 asked if subjects would choose the same appliance again. The responses were simplified into "yes/no" answers whereby "yes" was applied to patients who chose the same appliance and "no" was applied to those who chose the alternate appliance. Lastly, question 19 is a subjective evaluation of the overall quality of life during treatment with regard to teeth and orthodontic appliances. The answers were scored on a scale of $0-10$ whereby 0 indicates "vastly hindered" and 10 indicates "vastly improved."

The remaining questions corresponded to covariates which comprised demographic and other possible influential variables. They were compared between treatment groups. If a statistical difference existed between the two groups, the variable was adopted for a posteriori multivariable analysis. Logistic regressions for outcome variables were conducted with appropriate adjustments to account for potential effects. Several categorical variables were modified for convenience of analysis. The responses to questions $10 \mathrm{a}$ and $10 \mathrm{~b}$ were merged to quantify the number of unplanned appointments during treatment. The greater interval among emergency and urgent visits was selected for analysis. Similarly, questions 11 and 12 were combined into one variable documenting whether elastics or other auxiliaries were used during the course of treatment. The data was reduced to "yes" or "no" responses. 
Table 1

Survey questions used for analysis with corresponding variable abbreviations

\begin{tabular}{|c|c|c|}
\hline $\begin{array}{c}\text { Survey } \\
\text { Question }\end{array}$ & Question Summary & Variable Abbreviation \\
\hline 1 & Appliance type & APPLIANCE \\
\hline 3 & Previous treatment & PREVTX \\
\hline 6 & Primary reason for treatment & REASON \\
\hline 7 & Pre-treatment expectation(s) & EXPECT \\
\hline 8 & $\begin{array}{l}\text { Expected degree to which orthodontic objectives would } \\
\text { be fulfilled }\end{array}$ & FULFILLED \\
\hline $9 \mathrm{a}$ & Limit foods / Alter diet & FOODS \\
\hline $9 b$ & Difficulty chewing & CHEW \\
\hline $9 \mathrm{c}$ & Trouble swallowing & SWALLOW \\
\hline $9 \mathrm{~d}$ & Speech interference & SPEECH \\
\hline $9 \mathrm{e}$ & Content with look of appliance & LOOK \\
\hline $9 \mathrm{f}$ & Limit contact with others due to appliances & CONTACT \\
\hline $9 \mathrm{~g}$ & Comfortable in public with appliances & COMFORT \\
\hline $9 \mathrm{~h}$ & Worry concerning teeth or appliance & WORRY \\
\hline $9 \mathrm{i}$ & Self-conscious about appliance & SELF-CON \\
\hline$\overline{9 j}$ & Discomfort caused by appliance & DISCOMFORT \\
\hline $9 \mathrm{k}$ & Sensitive teeth & SENSITIVE \\
\hline 91 & Eat without discomfort & EAT \\
\hline $9 \mathrm{~m}$ & Use of medications for pain management & MEDS \\
\hline $10 \mathrm{a}$ and $10 \mathrm{~b}$ & Number of unplanned appointments & EMERG.URGENT \\
\hline 11 and 12 & Use of auxiliaries & ELASTICS.AUXILIARIES \\
\hline 13 & General health status & HEALTH \\
\hline 14 & Severity of discomfort during treatment & SEVERITY \\
\hline 15 & Happy with treatment outcome & OUTCOME \\
\hline 16 & Treatment objectives fulfilled & SATISFIED \\
\hline 17 & Propensity to choose same appliance again & OPINION \\
\hline 19 & $\begin{array}{c}\text { Quality of life related to teeth and appliance during } \\
\text { treatment }\end{array}$ & QUALITY \\
\hline
\end{tabular}




\section{CHAPTER III}

\section{RESULTS}

\section{A. Sample Characteristics}

The Invisalign treatment group and the control with fixed appliances were generally comparable for demographics and other covariates. Table 2 shows the distribution of sample demographics and other potential explanatory variables among the treatment groups. The treatment groups were commensurate with their distribution of age, sex, self-reported health status, prevalence of previous treatment, primary reason for treatment, degree of expected dental improvement, contentment with result, and unfulfilled treatment objectives. Although not reaching statistical significance in regard to primary reason for seeking orthodontic treatment, noticeably more Invisalign patients recorded "improve appearance" than did braces patients (72.5\% vs. 50\%). Figure 2 illustrates the primary reasons for seeking treatment. The braces sample reported a significantly higher number of unplanned office visits and more frequent use of auxiliaries. The responses in relation to pre-treatment expectations (question 7) were omitted from analysis. The instructions were to "check all that apply" which yielded an onerous number of combinations including several responses with less than 5 counts. Figure 3 summarizes the distribution of pre-treatment expectations in each group. Of particular interest is that more Invisalign patients cited straighter teeth $(97.5 \% \mathrm{vs.} 87 \%$ 
for braces) and esthetic appliance ( $87 \%$ vs. $13 \%$ for braces) as expectations in reference to their particular treatment and appliance system, respectively.

\section{Table 2}

Distribution of demographics and other covariates

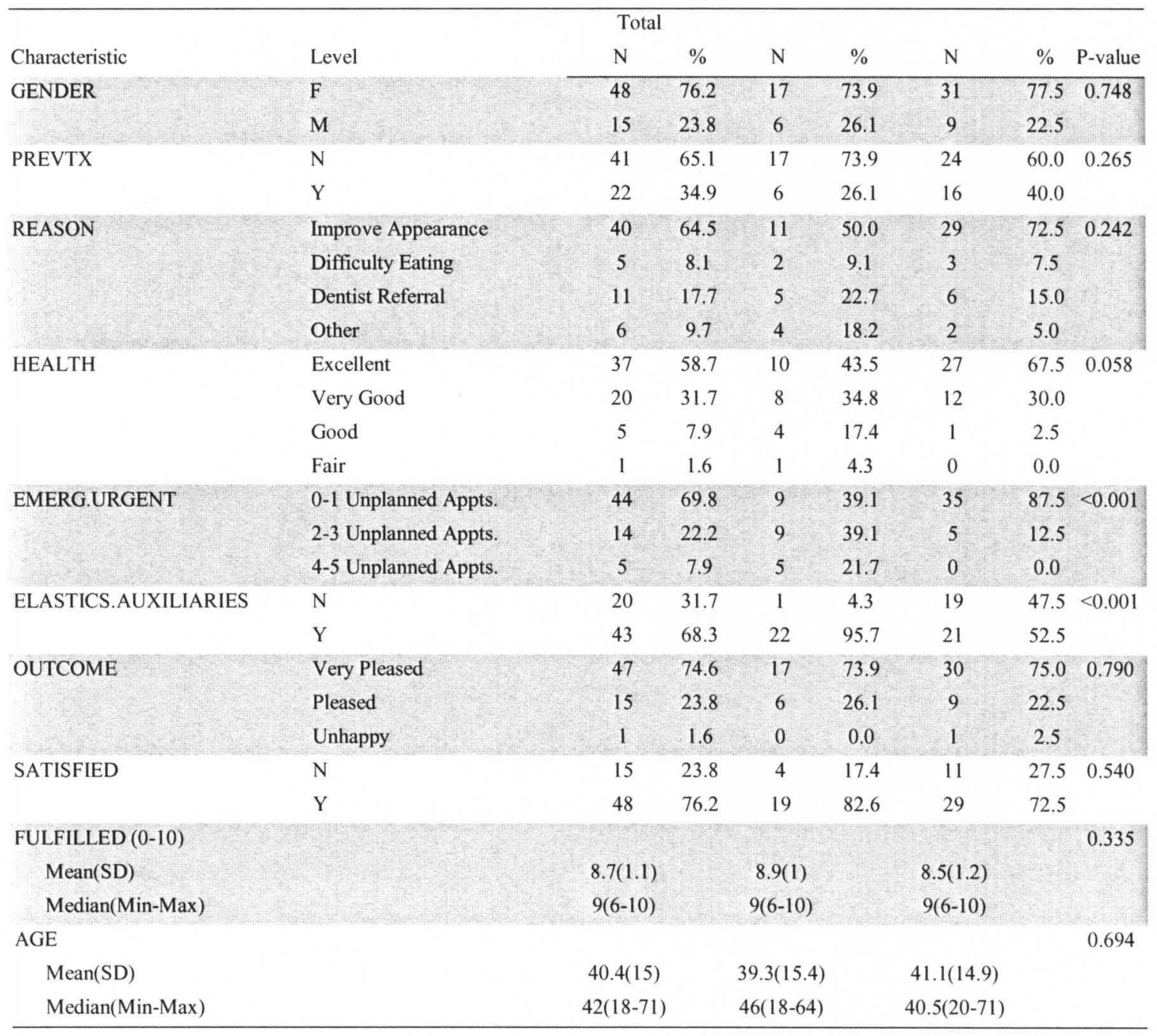

P-values from chi-squared or Fisher exact test. Variable levels with row counts less than 5 were excluded from statistical analysis. Variable "EXPECT" was not included in statistical analyses. 
Figure 2

Primary reasons for orthodontic treatment
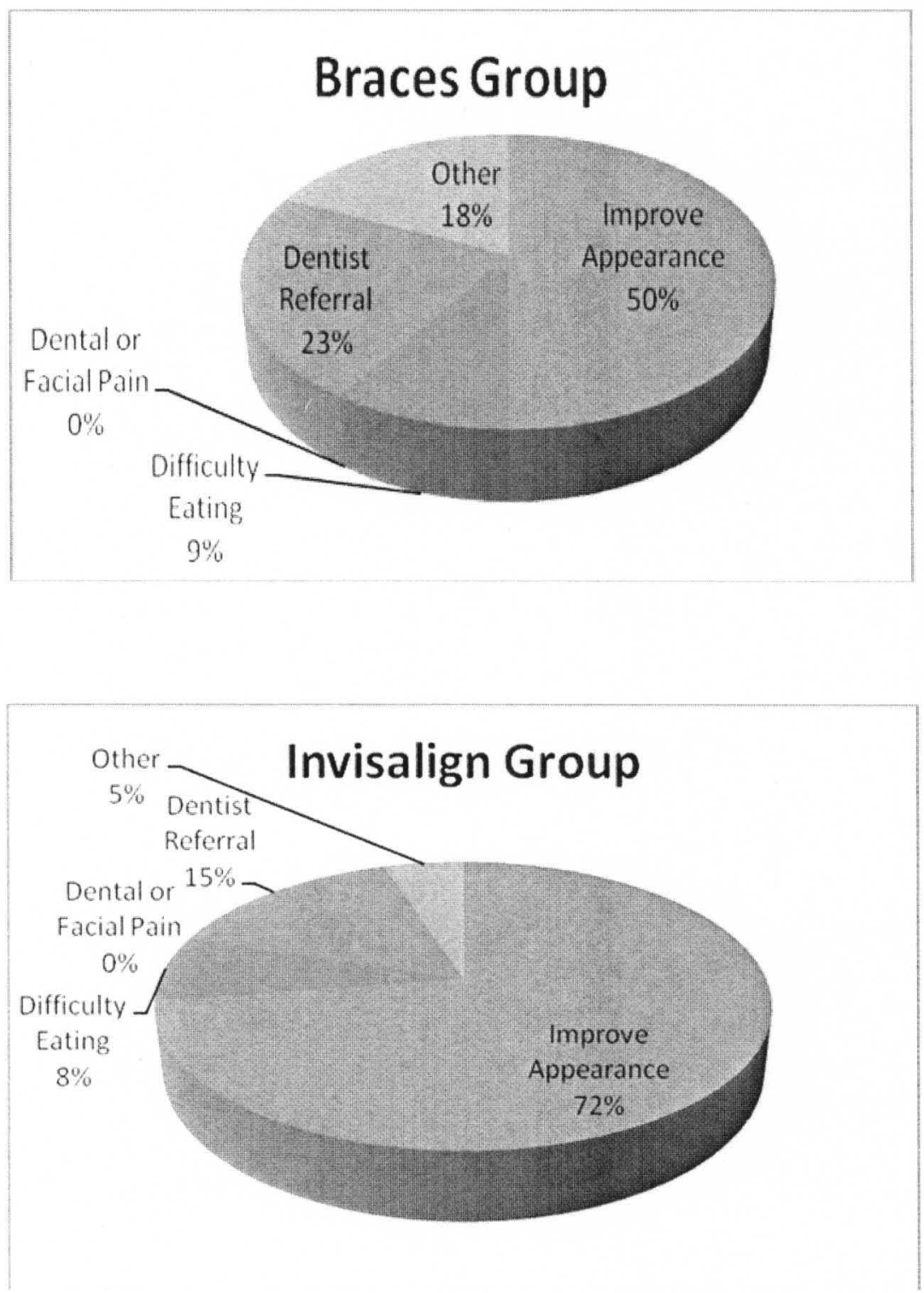


\section{Figure 3}

Pre-treatment expectations with regard to particular orthodontic appliances and treatment outcomes

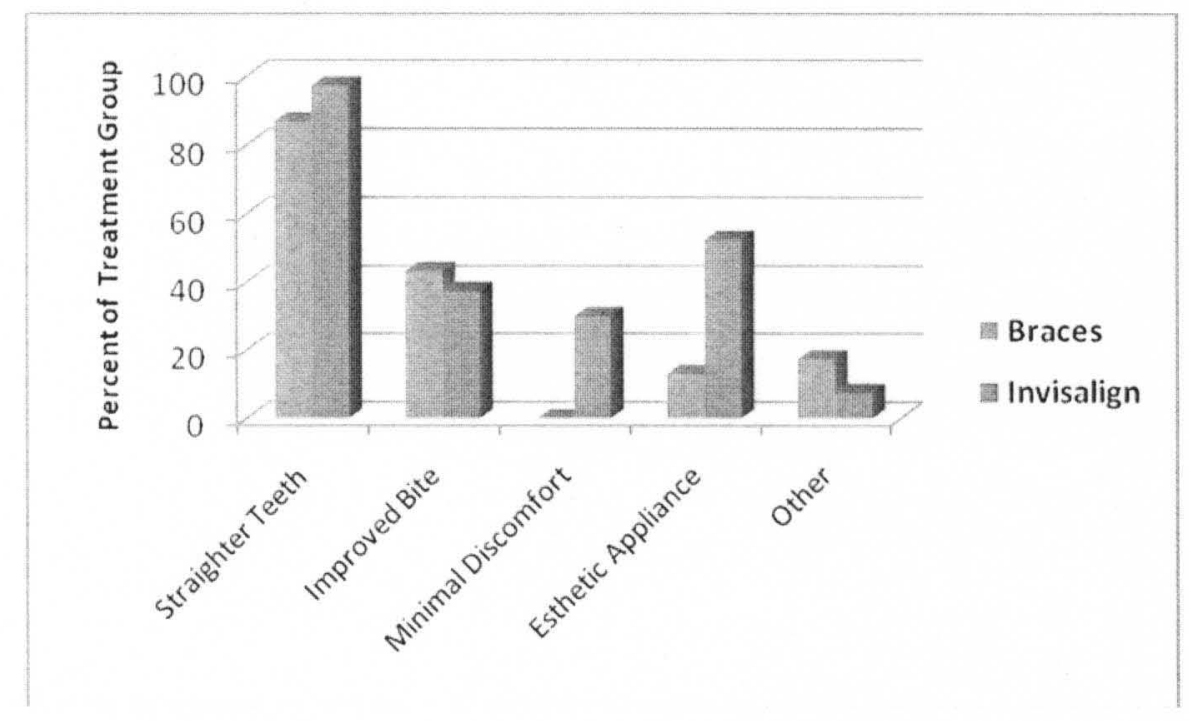

\section{B. Dependent Variables}

\section{Impact scores}

Tables 3 and 4 itemize the impact scores for the overall sample, braces group, and Invisalign group. The data is summarized with central tendency measures, mean and median, as well as distribution measures, standard deviation and range. A significant difference existed between the overall impact and the 3 subscales (functional, psychosocial, pain-related). The Invisalign group had unanimously higher scores for all 4 impact variables indicating a less negative response. Subjects' feedback was based on experience during the entire duration of treatment.

\section{Subjective measures of tolerance}


Tables 3 and 4 illustrate the subjective reports of treatment tolerance for the overall sample, braces group, and Invisalign group. The data from question 19 is summarized with central tendency measures, mean and median, as well as distribution measures, standard deviation and range. There was no significant difference between groups. The data from question 17 is summarized with distribution of responses. A statistically significant difference existed between the treatment groups. Invisalign exhibited a much higher preference for using the same appliance system if presented with the choice again (95\% vs. $60.9 \%$ for braces). The odds ratio was 11.7 with $95 \%$ confidence intervals (CI) of 2.1-124 (Table 5).

\section{Table 3}

\section{Outcome variables for overall sample}

\begin{tabular}{lccc} 
& \multicolumn{3}{c}{ Overall } \\
& Question & Mean(SD) & Median(Min-Max) \\
\cline { 3 - 4 } $\begin{array}{l}\text { Quality of Life } \\
\text { Impact }\end{array}$ & 19 & $7.9(2.2)$ & $9(2-10)$ \\
Overall & $9 \mathrm{a}-\mathrm{m}$ & $50.4(7.2)$ & $53(31-64)$ \\
Functional & $9 \mathrm{a}-\mathrm{d}$ & $15.4(2.9)$ & $16(8-20)$ \\
Pain & $9 \mathrm{j}-\mathrm{m}$ & $13.7(2.3)$ & $14(9-20)$ \\
Psych. & $9 \mathrm{e}-\mathrm{i}$ & $21.3(3.7)$ & $23(11-25)$ \\
Opinion & 17 & & 17.5 \\
No n(\%) & & 11 & 82.5 \\
Yes n(\%) & & 52 & \\
\hline
\end{tabular}

Abbreviations: Psych. - Psychosocial. 


\section{Table 4}

Outcome variables with regard to appliance system

\begin{tabular}{|c|c|c|c|c|c|c|}
\hline \multirow[b]{2}{*}{ Characteristic } & \multirow[b]{2}{*}{ Question } & \multicolumn{2}{|c|}{ Braces } & \multicolumn{2}{|c|}{ Invisalign } & \multirow[b]{2}{*}{ P-Value } \\
\hline & & $\operatorname{Mean}(\mathrm{SD})$ & Median(Min-Max) & Mean(SD) & Median(Min-Max) & \\
\hline Quality of Life & 19 & $8.1(2.1)$ & $8(4-10)$ & $7.8(2.3)$ & $9(2-10)$ & 0.604 \\
\hline \multicolumn{7}{|l|}{ Impact } \\
\hline Overall & $9 a-m$ & $44.9(7.3)$ & $43(31-57)$ & $53.6(4.9)$ & $54(42-64)$ & $<0.001$ \\
\hline Functional & $9 a-d$ & $13.3(2.8)$ & $13(8-17)$ & $16.7(2.1)$ & $17(11-20)$ & $<0.001$ \\
\hline Pain & $9 \mathrm{j}-\mathrm{m}$ & $12.5(2.1)$ & $13(9-15)$ & $14.4(2.2)$ & $14(10-20)$ & 0.002 \\
\hline Psych. & $9 e-i$ & $19.1(4.4)$ & $19(11-25)$ & $22.5(2.7)$ & $23(13-25)$ & 0.001 \\
\hline Opinion & 17 & & & & & 0.001 \\
\hline No $n(\%)$ & & 9 & 39.1 & 2 & 5 & \\
\hline Yes n(\%) & & 14 & 60.9 & 38 & 95 & \\
\hline
\end{tabular}

\section{Influence of covariates}

Because of the exploratory nature, the impact of numerous covariates was considered. Table 2 displays the covariates for the total sample, braces group, and Invisalign group. As previously mentioned, the number of either emergency or urgency appointments during treatment and the use of auxiliaries used during treatment were found to be associated with appliance group. The braces sample reported a significantly higher number of unplanned office visits and more frequent use of auxiliaries.

Unplanned appointments were highly and positively associated with braces ( $p$-value $<0.001)$, with an odds ratio (OR) for B (2-3 appointments) vs. A (0 to 1 appointments) of 6.6 with $95 \%$ CI of 1.8-27.3 (Table 5). The use of auxiliaries was also highly positively associated with braces ( $\mathrm{p}$-value $<0.001$ ) featuring an OR of 17.1 with $95 \%$ CI of 3.1-435 (Table 5). These two adjustments were subsequently included in logistic regression 
multivariable analyses for the dependent variables. P-values for overall, functional, painrelated, and psychosocial impacts after adjusting for unplanned appointments and auxiliaries were $0.016,0.034,0.173,0.025$, respectively (Table 6 ). Therefore in general, after multiple adjustment, the significance of these variables seems to be preserved, with the possible exception of pain.

\section{Table 5}

$\underline{\text { Odds ratios }(\mathrm{OR}) \text { for adjustment and outcome variables }}$

\begin{tabular}{lcc}
\hline & OR & CI \\
\cline { 2 - 3 } Adjustment Variables & & \\
EMERG.URGENT & 1 & - \\
0-1 Appts. & 6.6 & $1.8-27.3$ \\
2-3 Appts. & Inf & $2.8-\operatorname{lnf}$ \\
4-5 Appts. & & \\
ELASTICS.AUXILIARIES & & - \\
No & 1 & $3.1-435$ \\
Yes & & \\
Outcome Variable & & \\
OPINION & 1 & - \\
No & 11.7 & $2.1-124$ \\
Yes &
\end{tabular}

For Adjustment Variables, OR is wrt Braces vs Invisalign. For Outcome Variable, OR is wit Invisalign compared to braces. For all three variables, the top level (A for EMERG.URGENT, "No" for ELASTICS.AUXILIARIES, "No" for Opinion, is the reference level, which is why the OR is 1 with no Confidence Intervals. 


\section{Table 6}

Multivariable analyses adjusted for unplanned visit and auxiliaries

\begin{tabular}{|l|c|c|}
\hline & $\begin{array}{c}\text { P-value } \\
\text { (Pre-adjustment) }\end{array}$ & $\begin{array}{c}\text { P-value } \\
\text { (Post-adjustment) }\end{array}$ \\
\hline Impact & & \\
\hline Overall & $<0.001$ & 0.016 \\
\hline Function & $<0.001$ & 0.034 \\
\hline Pain & 0.002 & 0.173 \\
\hline Psych. & 0.001 & 0.025 \\
\hline
\end{tabular}

Abbreviations: Psych. - Psychosocial; P-Values calculated using wilcoxon exact test or t-test (unequal variances) 


\section{CHAPTER IV}

\section{DISCUSSION}

Invisalign therapy is an esthetic alternative to conventional appliances and it is a growing area of orthodontics. Excitement surrounding this technology has overshadowed the research behind it. It is imperative to validate information disseminated to the public. It has been purported to minimize side effects associated with treatment. Clear aligner studies show promising results with regard to esthetics, improved periodontal health, and increasing efficacy. The presumption that Invisalign is better tolerated by patients has been promulgated by marketing campaigns and providers alike yet there is only preliminary evidence to date. The preponderance of literature comparing patient tolerance of removable and fixed modalities has used removable appliances that faintly resemble current clear aligners. Examples include removable plates and functional appliances which carry little relevance to Invisalign trays. The most compatible study compared the impacts of Invisalign and fixed appliances using a daily diary for the first week of treatment. Miller et al (2009) found Invisalign to perform better during this abbreviated time period. In the present study, impacts and subjective tolerance were examined as a reflection of the entire course of treatment. It has been proposed that contemporaneous daily assessments are more reliable and accurate than retrospective recalls. Nonetheless, a retrospective account was deliberately used to best characterize the patient response during the entire course of treatment rather than a day snapshot or 
week-long excerpt. The findings from this study corroborate previous research that examined treatment effects over first week only. Efforts were taken to control for nuances between patients such as expectations, objectives, and satisfaction of treatment. These can be influential in subjective responses as witnessed by Sergl et al (2000) who found that subjects perceived severity of malocclusion was inversely related to report of discomfort.

Negative treatment impacts were consistently less severe with the Invisalign group than the braces group. This was true for overall impacts and individually for all 3 subcomponents (functional, psychosocial, pain-related). This suggests that Invisalign imposes less discomfort and it is less disruptive to a patient's daily routine. Related prospective studies have concluded that patients adapt to the encumbrances and discomfort imparted by orthodontic appliances. They claimed that differences between appliance systems waned as adaptation occurred. These findings are challenged by the present study since it embodies the entire duration of treatment and a difference persisted. Anticipated discomfort and social embarrassment with adult orthodontics cause enough despair to prevent prospective patients from pursuing treatment. The reduced negative impacts illustrated in this study portends Invisalign is better tolerated by adult patients. This may be contributing to the increasing number of adult patients witnessed in recent years.

The prediction that Invisalign is better tolerated was tested in the study and it yielded contradictory results. No difference was discerned between treatment groups regarding the patients' subjective report on overall quality of life related to their teeth and/or orthodontic appliances. To the contrary, there was a remarkable difference in 
patients' predilection to choose the same appliance if granted the choice again. There are several plausible explanations. For example, Invisalign patients, despite their experience, may be reluctant to use traditional braces due to esthetic concerns. Esthetic (inconspicuous) appliance was cited as a reason for choosing clear trays in $52.5 \%$ of the Invisalign sample which manifests the esthetic imperative. Perhaps an esthetic option would not be forsaken regardless of patients' experience with the modality, even if it was negative. For comparison, only $13 \%$ of braces patients reported esthetic appliances as a reason for choosing that particular modality. The conflicting result between the two subjective outcome measures furnishes the complexity of defining and calculating patient tolerance. Much is concealed with individual nuances. For example, some patients have a proclivity to respond more positively or negatively, depending on their demeanor. Information was collected to control for confounding influences and this was comprehensive but not exhaustive. Each covariate was carefully chosen based on sound theory or shrewd speculation. The sample groups were largely comparable for these variables. The sample was entirely extracted from private practices in the same geographic region and practitioners were experienced with both treatment modalities. The two variables that were not comparable between the two groups were subsequently used in a multivariable analysis to adjust for possible effects.

In large, the results were unaffected when adjustments were made for the confounders, unplanned visits and use of auxiliaries. Only the pain-related subcomponent of the negative impacts lost significance. With regards to unplanned visits, one possible explanation is that pain is often the reason for these appointments, implying that pain and unplanned visits are intimately related. This introduces 
multicollinearity to the model which weakens the ability to discern the specific influence of each predictor variable. Ultimately, the reduced significance of pain should not be rashly interpreted as meaning there was no difference between the two groups. Rather, multicollinearity may have tainted the statistics. In reference to auxiliaries, these may have a profound impact on discomfort and could have biased the two treatment groups since they were prescribed more frequently among braces patients. The effect of auxiliaries is undeniable as they can produce discomfort and place a burden on patients with compliance. Thus, the difference in pain-related impacts may essentially be minimal or absent if both treatment groups used auxiliaries with the same frequency. Results must be interpreted with caution.

This study was edifying and underscores the multifactorial nature of patient response to orthodontic appliances and treatment. Further efforts could be made to strengthen current conclusions. Increasing sample size would ostensibly attenuate these flaws by introducing more power into the study. This in turn reduces type II error which increases the chance of detecting potential differences between groups if they truly exist. Perhaps there were some mild differences in other confounding variables that were not adjusted for in the logistic regression. If so, these variables could be included in a future statistical model. As previously discussed, pre-treatment expectations were omitted from analysis due to low counts with certain answer choices. This may be attributed to questions design which was, "check all that apply." Figure 3 shows that motivations were disparate between two treatment groups. Different pre-treatment expectations could potentially influence reports on pain and other impacts. Increasing survey responses would also lend to a more stringent inclusion/exclusion criteria thereby better controlling 
for confounders. For example, orthognathic surgery patients could have been eliminated from study. Furthermore, the study may benefit from randomization; however, it may be difficult to randomize subjects due to demanding esthetic concerns or case complexity. As previously mentioned, some patients may have a strong propensity toward Invisalign due to an esthetic imperative and be resistant to fixed appliances. Conversely, most literature to date favors fixed appliances when comparing treatment efficacies so patients with complex treatment goals may be less inclined to pursue Invisalign therapy. These factors pose a challenge to truly randomize patients. To that effect, the precise manner in which patients from this study were assigned to their treatment group is nebulous and each clinician may be different in their case presentations. It is assumed that patients were given informed consent with unbiased information. The sample would lose its randomization if patients were persuaded to use a specific modality based on media propaganda. Also, each clinician may be able to fulfill treatment goals with either appliance system but simply has a preference for one modality and this may have been imparted, to some extent, on the patient's decision. These are speculative but could have contributed some bias to sample distributions. Ultimately, the results may be improved by increasing sample size and improving its randomization.

Further improvement may result from the inclusion of additional variables. Two specific variables that were not controlled in present study are initial case complexity and the finished result. Both of these may bear influence on outcome variables. There are numerous indices used to quantify case complexity such as the initial Peer Assessment Review (PAR) score and ABO Discrepancy Index (DI) score. Either of these could be used to characterize case difficulty between two groups. Likewise, treatment efficacy 
could be evaluated based on the final PAR score ABO Objective Grading Score (OGS). It is logical to presume that the treatment outcome might influence overall satisfaction with treatment and particular appliance. Their effect would likely be modulated by treatment expectations, which was included in the current study, but it would still be worth exploring the impact of treatment outcomes.

Another avenue to improve study is to amend question design. In general, numeric variables have more conclusive power than categorical or interval variables. Unfortunately, some questions fundamentally cannot have numeric responses such as, "what was primary reason for seeking treatment?" Also, the retrospective nature poses some difficulty. The accuracy of numeric answers may be dubious if they are a reflection on something that occurred 1-2 years ago. Future survey studies could be prospective in nature. In this design, the surveys would be distributed at numerous, random intervals throughout treatment and completed immediately in the format of daily diary. This would have the benefit of encompassing the entire treatment time and it would remedy the problems with reliability of retrospective recall. 


\section{CHAPTER V}

\section{SUMMARY AND CONCLUSIONS}

\section{A. Summary}

In sum, the results were favorable for Invisalign. Invisalign provokes less negative impacts related to treatment than fixed appliances. However, it is interesting that the two subjective reports on patient tolerance of their orthodontic appliance had contradictory results. This underscores the complex nature of predicting and quantifying individual responses to treatment. The results provide a scientific foundation to the nuances between various orthodontic appliances. On the macro scale, this information can be disseminated to the public since media platforms have already been exploited. On the micro scale, this knowledge can be used as an adjunct during patient consultations. Regardless of which forum, these findings should improve patient education. Orthodontic professionals are public fiduciaries who are entrusted to provide evidencedbased information to patients and guide them in selecting the appropriate appliance system.

\section{B. Conclusions}

1. In comparison to the fixed appliance group, Invisalign patients experienced less negative impacts with regards to teeth and orthodontic appliances over the course of treatment. This was true for the overall impact and individually for the functional, pain-related, and psychosocial subscales. Even after multiple 
adjustments, the significance of these differences was preserved, with the possible exception of the pain-related subcomponent.

2. There were no differences among treatment groups with subjective reports on the overall quality of life regarding their teeth and appliances during orthodontic treatment.

3. Invisalign patients are more likely to choose the same appliance system, if presented with the choice again.

4. Clearly, Invisalign straightens teeth. 


\section{REFERENCES}

American Association of Orthodontists (AAO) website. www.braces.org. 2011.

Align Technology, Inc. Invisalign ${ }^{\circledR}$ website. www.invisalign.com

Atchison K, Dolan TA. Development of the geriatric oral health assessment index. J Dent Educ 1990;54:680-7.

Boyd RL, Miller RJ, Vlaskalic V. The Invisalign system in adult orthodontics: mild crowding and space closure cases. J Clin Orthod 2000;34:203-12.

Breece GL, Nieberg LG. Motivations for adult orthodontic treatment. J Clin Orthod. 1986 Mar;20(3):166-71.

Burgersdijk R, Truin GJ, Frankenmolen F, Kalsbeek H, van't Hof M, Mulder J. Malocclusion and orthodontic treatment need of 15-74-year-old Dutch adults. Community Dent Oral Epidemiol 1991;19:64-7.

Buttke TM, Proffit WR. Referring adult patients for orthodontic treatment. J Am Dent Assoc 1999;130:73-9.

Djeu G, Shelton C, Maganzini A. Outcome assessment of Invisalign and traditional orthodontic treatment compared with the American Board of Orthodontics objective grading system. Am J Orthod Dentofacial Orthop 2005;128:292-8.

Fritz U, Diedrich P, Wiechmann D. Lingual technique - patients' characteristics, motivation, and acceptance. Interpretation of a retrospective survey. $\mathbf{J}$ Orofac Orthop 2002;63:228-33.

Giancotti A, Di Girolamo R. Treatment of severe maxillary crowding using Invisalign and fixed appliances. J Clin Orthod 2009;43:583-9.

Giancotti A, Mampieri G, Greco M. Correction of deep bite in adults using the Invisalign system. J Clin Orthod 2008;42:719-26. 
Gazit-Rappaport T, Haisraeli-Shalish M, Gazit E. Psychosocial reward of orthodontic treatment in adult patients. Eur J Orthod 2010 Aug;32(4):4416.

Hohoff A, Fillion D, Stamm T, et al. Oral comfort, function and hygiene in patients with lingual brackets. A prospective longitudinal study. J Orofac Orthop 2003; 64:359-71.

Hohoff A, Seifert E, Fillion D, et al. Speech performance in lingual orthodontic patients measured by sonography and auditative analysis. Am J Orthod Dentofacial Orthop 2003; 123:246-52.

Honn M, Goz G. A premolar extraction case using the Invisalign system. J Orofac Orthop 2006;67:385-94.

Kesling HD. The philosophy of tooth positioning appliance. Am J Orthod 1945; 31:297-304.

Kravitz ND, Kusnoto B, Agran B, Viana G. Influence of attachments and interproximal reduction on the accuracy of canine rotation with Invisalign. Angle Orthod 2008;78:682-7.

Kravitz ND, Kusnoto B, BeGole E, Obrez A, Agran B. How well does Invisalign work? A prospective clinical study evaluating the efficacy of tooth movement. Am J Orthod Dentofacial Orthop 2009;135:27-35.

Kuo E, Miller R. Automated custom-manufacturing technology in orthodontics. Am J Orthod Dentofacial Orthop 2003;123:578-81.

Lagravere MO, and Flores-Mir C. The treatment effects of Invisalign orthodontic aligners: a systematic review. J Am Dent Assoc 2005;136:1724-9.

Lew KK. Attitudes and perceptions of adults towards orthodontic treatment in an Asian community. Community Dent Oral Epidemiol. 1993 Feb;21(1):315.

Meier B, Wiemer KB, Miethke RR. Invisalign patient profiling. Analysis of a prospective survey. J Orofac Orthop 2003 Sep;64(5):352-8.

Miethke RR, Vogt S. A comparison of the periodontal health of patients during treatment with Invisalign system and with fixed orthodontic appliances. J Orofac Orthop 2005;66(3):219-29. 
Miethke RR, Brauner. A comparison of the periodontal health of patients during treatment with Invisalign system and with fixed lingual orthodontic appliances. J Orofac Orthop 2007;68:223-31.

Miller KB, McGorray SP, Womack R, Quintero JC, Perelmuter M, Gibson J, et al. A comparison of treatment impacts between Invisalign aligner and fixed appliance therapy during first week of treatment. Am J Orthod Dentofacial Orthop 2007;131:302.e1-9.

Nedwed V, Miethke RR. Motivation, acceptance, and problems of Invisalign patients. J Orofac Orthop 2005;66:162-73.

Norris RA, Brandt DJ, Crawford CH, Fallah M. Restorative and Invisalign: a new approach. J Esthet Restor Dent. 2002;14(4):217-24.

Proffit WR, Fields HW, Moray LJ. Prevalence of malocclusion and orthodontic treatment need in the United States: Estimates from the NHANES III survey. In J Adult Orthod Orthognath Surg Vol. 13, No. 2, 1998.

Proffit WR, Field, HW. Sarver, DM. Contemporary Orthodontics: Fourth Edition. Mosby. 2007.

Rosvall MD, Fields HW, Ziuchkovski J, Rosenstiel SF, Johnston WM. Attractiveness, acceptability, and value of orthodontic appliances. Am J Orthod Dentofacial Orthop 2009;135:276.e1-276.e12.

Salonen L, Mohlin B, Gotzlinger B, Hellden L. Need and demand for orthodontic treatment in an adult Swedish population. Eur J Orthod 1992;14:359-68.

Schaefer I, Braumann B. Halitosis, oral health and quality of life during treatment with Invisalign( $(\mathbb{R})$ and the effect of a low-dose chlorhexidine solution. J Orofac Orthop. 2010 Nov;71(6):430-41. Epub 2010 Nov 17.

Schupp W, Haubrich J, Neumann I. Class II correction with the Invisalign system. J Clin Orthod 2010;44:28-35.

Searcy VL, Chisick MC. Perceived, desired, and normatively determined orthodontic treatment needs in male US Army recruits. Community Dent Oral Epidemiol. 1994 Dec;22(6):437-40.

Sergl HG, Klages U, Zentner. Pain and discomfort during orthodontic treatment: causative factors and effects on compliance. Am J Orthod Dentofacial Orthop 1998;114(6):684-91. 
Sergl HG, Klages U, Zentner A. Functional and social discomfort during orthodontic treatment - effects on compliance and prediction of patients' adaptation by personality. Eur J Orthod 2000;22(3):307-15.

Stevnik A, Espeland L, Linge BO, Linge L. Lay attitudes to dental appearance and need for orthodontic treatment. Eur J Orthod 1997; 19:271-7.

Stewart FN, Kerr JS, Taylor PJ. Appliance wear: the patient's point of view. Eur J Orthod (1997) 19 (4): 377-382.

Vardimon AD, Robbins D, Brosh T. In-vivo von Mises strains during Invisalign treatment. Am J Orthod Dentofacial Orthop 2010;138:399-409.

Vincent S. Evaluation of Invisalign treatment utilizing the American Board of Orthodontics Objective Grading System for dental casts. Am J Orthod Dentofacial Orthop 2005; 127:268-9.

Whitesides J, Pajewski NM, Bradley TG, Iacopino AM, Okunseri C. Sociodemographics of adult orthodontic visits in the United States. Am J Orthod Dentofacial Orthop. 2008 Apr;133(4):489.e9-14.

Womack WR. Four-premolar extraction treatment with the Invisalign system. J Clin Orthod 2006;40:493-500.

Womack WR, Day RH. Surgical-orthodontic treatment using the Invisalign system. J Clin Orthod 2008;42:237-45.

Wong B. Invisalign A to Z. Am J Orthod Dentofacial Orthop 2002;121:540-1.

Ziuchkovski JP, Fields HW, Johnston WM, Lindsey DT. Assessment of perceived orthodontic appliance attractiveness. Am J Orthod Dentofacial Orthop 2008;133:S68-78. 
APPENDIX 
Appendix A

\title{
Figure 1
}

\section{Survey Instrument}

INSTRUCTIONS: Thank you in advance for completing the following survey about how your orthodontic appliances (Invisalign trays or braces) affected your life throughout the course of treatment. To the best of your knowledge, answer only what you felt and experienced while you were in treatment, not what you think is the right answer. There are no right or wrong answers to these questions.

1. What type of orthodontic appliances did you have most recently as an adult?

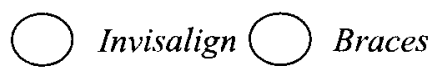

2. How long ago did you finish your most recent orthodontic treatment as an adult?

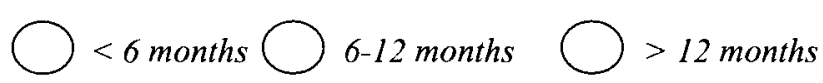

3. Did you have previous orthodontic treatment prior to your most recent phase of treatment? If yes, please answer questions 4 and 5. If no, please skip to question 6.

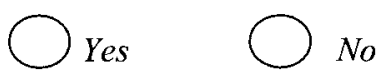

4. If yes to question 3, what type of orthodontic appliances did you previously wear?

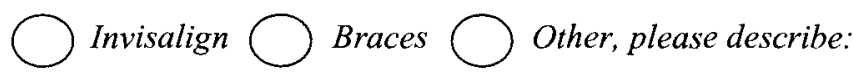

5. In reference to question 4 , how long ago did you finish your previous orthodontic treatment?

$$
\bigcirc<3 \text { years } \bigcirc 3-7 \text { years } \bigcirc>7 \text { years }
$$

NOTE: Please answer the remaining questions based on your experience with your most recent orthodontic treatment, not regarding any prior orthodontic treatment. Thank you.

6. What was the primary reason for seeking your orthodontic treatment? (Please check one)

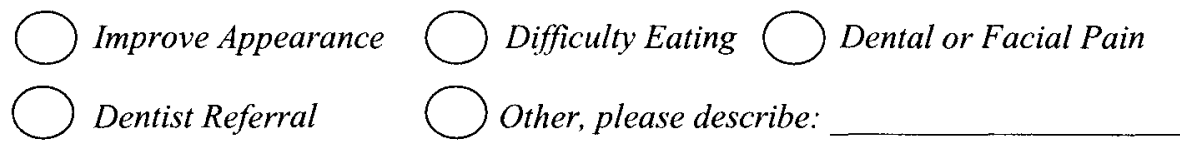

7. Which of the following describe your pre-treatment expectations with regard to your orthodontic appliances and treatment? (Please check all that apply) $\bigcirc$ Straighter Teeth $\bigcirc$ Improved Bite
Esthetic (Inconspicuous) Appliances

\author{
Minimal Discomfort \\ Other, please describe:
}


8. Prior to treatment, to what degree did you expect your orthodontic treatment objectives to be fulfilled?

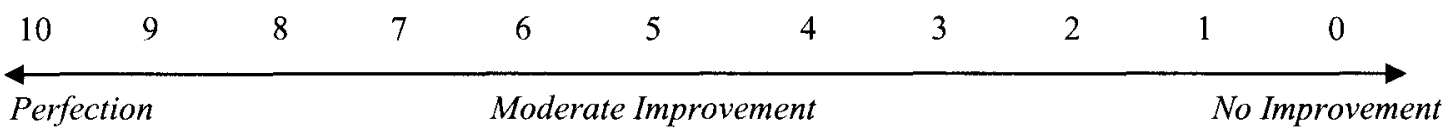

9. Please check one response for each of the following questions.

\begin{tabular}{|l|l|l|l|l|l|}
\hline $\begin{array}{l}\text { During your orthodontic treatment, how } \\
\text { often: }\end{array}$ & Always & Often & Sometimes & Seldom & Never \\
\hline $\begin{array}{l}\text { a. Did you limit the kinds or amounts of } \\
\text { food you ate because of your } \\
\text { orthodontic appliances or because of } \\
\text { problems with your mouth or teeth? }\end{array}$ & & & & & \\
\hline $\begin{array}{l}\text { b. Did you have trouble biting or } \\
\text { chewing any kinds of foods, such as } \\
\text { meat or apples? }\end{array}$ & & & & & \\
\hline $\begin{array}{l}\text { c. Were you able to swallow } \\
\text { comfortably? }\end{array}$ & & & & & \\
\hline $\begin{array}{l}\text { d. Did your orthodontic appliances } \\
\text { interfere with your speech? }\end{array}$ & & & & & \\
\hline $\begin{array}{l}\text { e. Were you pleased or happy with the } \\
\text { look of your orthodontic appliances? }\end{array}$ & & & & & \\
\hline $\begin{array}{l}\text { f. Did you limit contact with people } \\
\text { because of the appearance of your } \\
\text { orthodontic appliances? }\end{array}$ & & & & & \\
\hline $\begin{array}{l}\text { g. Did you feel uncomfortable eating in } \\
\text { front of people because of problems } \\
\text { with your orthodontic appliances? } \\
\text { tongue }\end{array}$ & & & & & \\
\hline $\begin{array}{l}\text { here you worried or concerned about } \\
\text { orthodontic appliances? } \\
\text { orthodontic appliances? }\end{array}$ & & & & & \\
\hline j. Did your orthodontic appliances cause & & & & & \\
\hline
\end{tabular}




\begin{tabular}{|l|l|l|l|l|l|}
\hline $\begin{array}{l}\text { k. Were your teeth sensitive to hot, cold, } \\
\text { or sweets? }\end{array}$ & & & & & \\
\hline $\begin{array}{l}\text { l. Were you able to eat without feeling } \\
\text { discomfort? }\end{array}$ & & & & & \\
\hline $\begin{array}{l}\text { m. Did you use medication to manage } \\
\text { pain or discomfort related to your } \\
\text { orthodontic appliances? }\end{array}$ & & & & & \\
\hline
\end{tabular}

10. Please check one response for each of the following questions.

\begin{tabular}{|l|l|l|l|l|l|}
\hline During your treatment, approximately how many times did you: & $\mathbf{0 - 1}$ & $\mathbf{2 - 3}$ & $\mathbf{4 - 5}$ & $\mathbf{6 - 7}$ & Over 7 \\
\hline $\begin{array}{l}\text { a. Schedule an appointment on the same day for an emergency } \\
\text { situation such as a broken appliance, injury, or major concern? }\end{array}$ & & & & & \\
\hline $\begin{array}{l}\text { b. Schedule an appointment within a few days for an urgent situation } \\
\text { such as a broken appliance, injury, or major concern? }\end{array}$ & & & & & \\
\hline
\end{tabular}

11. Did you use rubber bands (elastics) as part of your treatment?<smiles></smiles>

12. What other auxiliaries did you use during your treatment? (Please check all that apply)

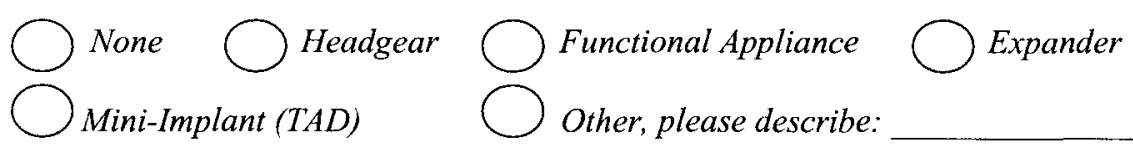

13. How would you best describe your general health status during your orthodontic treatment?

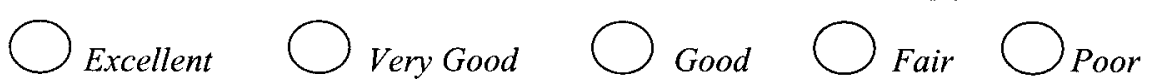

14. Please check the one choice that best describes the severity of discomfort over the course of your treatment.

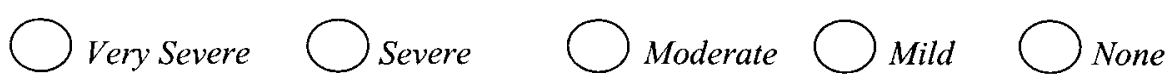

15. In summary, how pleased are you with outcome of treatment? (Please check one)
$\bigcirc$
Very pleased
Pleased
$\bigcirc$ Neutral
Unhappy
Very Unhappy

16. Relative to your pre-treatment expectations, which specific objectives were not fulfilled with your orthodontic appliances and treatment? (Please check all that apply)

\}$_{\text {Nreatment Length }}^{\text {Straight Teeth }}$ Osthetics of Appliances $\bigcirc$ Improved Bite $\bigcirc$ Minimal Discomfort 
17. Please check the one response that best reflects your opinion.

\begin{tabular}{|l|l|}
\hline I had traditional braces and I would choose traditional braces again. \\
\hline & I had Invisalign trays and I would choose Invisalign again. \\
\hline I had traditional braces and I would choose Invisalign if I had to choose again. \\
\hline I had Invisalign and I would choose traditional braces if I had to choose again. \\
\hline
\end{tabular}

18. Did you have any other problems or concerns about your teeth or orthodontic appliances since the inception of your orthodontic treatment? If so, please describe.

19. Results aside, how would you rate your overall quality of life with regard to your teeth and/or orthodontic appliances during the entire course of your treatment? (Please circle one number)

$\begin{array}{ccccccccccc}10 & 9 & 8 & 7 & 6 & 5 & 4 & 3 & 2 & 1 & 0 \\ \underset{\text { Vastly Improved }}{\longrightarrow} & \text { Unaffected }\end{array}$




\section{Appendix B}

To whom it may concern,

We are the owners and custodians of the records for patients in our practice. We are working with Dr. Keith C Nicholson of the University of Louisville to complete a research project. Our patient records will be made available to Dr. Nicholson to complete his research project. Should any additional information be needed from us, kindly contact us by email at dgerman@germanburke-ortho.com or by telephone at 937-4266860 .

Sincerely,

Daniel S. German and Stephen P. Burke 
Appendix C

For IRB Approval Stamp

Title: A survey study comparing adult orthodontic patient quality of life between Invisalign and fixed appliance therapy

Date: $12 / 17 / 10$

Dear (prospective subject):

You are being invited to participate in a research study by answering the attached survey about adult patient quality of life during orthodontic treatment pertaining to oral function and discomfort. If you are willing to participate then please complete the enclosed survey and mail it in the stamped and addressed envelope that is provided. There are no known risks for your participation in this research study. The information collected may not benefit you directly. The information learned in this study may be helpful to others. The information you provide will enlighten orthodontists with valuable insight regarding advantages and limitations associated with various orthodontic appliances. This will guide orthodontists in designing treatment for future patients. Your completed survey will be stored at University of Louisville Department of Orthodontics. The survey will take approximately 5 minutes to complete.

Individuals from the University of Louisville Department of Orthodontics, the Institutional Review Board (IRB), the Human Subjects Protection Program Office (HSPPO), and other regulatory agencies may inspect these records. In all other respects, however, the data will be held in confidence to the extent permitted by law. Should the data be published, your identity will not be disclosed.

Taking part in this study is voluntary. By completing this survey you agree to take part in this research study. You do not have to answer any questions that make you uncomfortable. You may choose not to take part at all. If you decide to be in this study you may stop taking part at any time. If you decide not to be in this study or if you stop taking part at any time, you will not lose any benefits for which you may qualify. 
If you have any questions, concerns, or complaints about the research study, please contact:

\section{Dr. Keith Nicholson, University of Louisville Orthodontic Resident} (919)923-4725

If you have any questions about your rights as a research subject, you may call the Human Subjects Protection Program Office at (502) 852-5188. You can discuss any questions about your rights as a research subject, in private, with a member of the Institutional Review Board (IRB). You may also call this number if you have other questions about the research, and you cannot reach the study doctor, or want to talk to someone else. The IRB is an independent committee made up of people from the University community, staff of the institutions, as well as people from the community not connected with these institutions. The IRB has reviewed this research study.

If you have concerns or complaints about the research or research staff and you do not wish to give your name, you may call 1-877-852-1167. This is a 24 hour hot line answered by people who do not work at the University of Louisville.

Sincerely,

Keith Nicholson, DDS 


\title{
CURRICULUM VITAE
}

\author{
Keith C. Nicholson
}

331 Cannons Lane $\cdot$ Louisville, KY $40206 \cdot$ (919) 923-4725 • kenicholson13@yahoo.com

\section{EDUCATION}

University of Louisville, Louisville, $\mathrm{KY}$

June 2011

(Candidate)

Certificate in Orthodontics

Master of Science in Oral Biology

University of North Carolina, Chapel Hill, NC

June 2009

Certificate in Advanced Education in General Dentistry

University of North Carolina, Chapel Hill, NC

May 2008

DDS Honors with Distinction

Davidson College, Davidson, $\mathrm{NC}$

BA Economics magna cum laude

NCAA Division I Soccer Scholarship

C.F. Myers Jr. - Burlington Honors Scholarship

\section{LICENSURE/CERTIFICATION}

North Carolina State Board Dental License

Kentucky State Board Student Limited Dental License

Invisalign Certification

Charles H. Tweed Orthodontic Course Certification

Council of Interstate Testing Agency Certification

May 2004

\section{CLINICAL EXPERIENCE}

Proficient with .018 and .022 appliances, self-ligating appliances, functional appliances, combined surgical treatment, TAD placement, diode laser, Invisalign, Cone Beam CT

\section{RESEARCH / PRESENTATIONS}

Comparison of adult orthodontic patient quality of life between Invisalign and fixed appliance therapy Master's thesis project (in progress)

A finite element analysis of traumatic fractures in a maxillary central incisor Thesis for DDS Honors with Distinction, May 2008

Poster presentation at the 2009 IADR/AADR meeting in Miami, FL

\section{TEACHING EXPERIENCE}

University of Louisville Resident Instructor

2008 - present

2009 - present

2009 - present

September 2010

April 2008

Composed and delivered lectures for DMD orthodontic courses

Instructed DMD students on orthodontic appliances in laboratory setting

PROFESSIONAL AFFILIATIONS

American Association of Orthodontists 
American Dental Association

\section{VOLUNTEER SERVICE}

Cleft Palate Gallop 5K - Founder, Event Coordinator, Volunteer

2008 - present

Davidson College Career Services Mentor

2006 - present

\section{EXTERNSHIPS}

UNC Hospitals General Practice Residency

September 2007

Peak Vista Community Dental Clinic

July 2007

\section{HOBBIES}

Soccer, Tennis, Reading, Running 